\title{
BioCTS2012
}

NISTIR 7877

\author{
BioCTS 2012: \\ Advanced Conformance Test \\ Architectures and Test Suites for \\ Biometric Data Interchange Formats and \\ Biometric Information Records
}

Fernando L. Podio

Dylan Yaga

Christofer J. McGinnis 
This page left intentionally blank 


\section{BioCTS 2012: Advanced Conformance Test Architectures and Test Suites for Biometric Data Interchange Formats and Biometric Information Records}

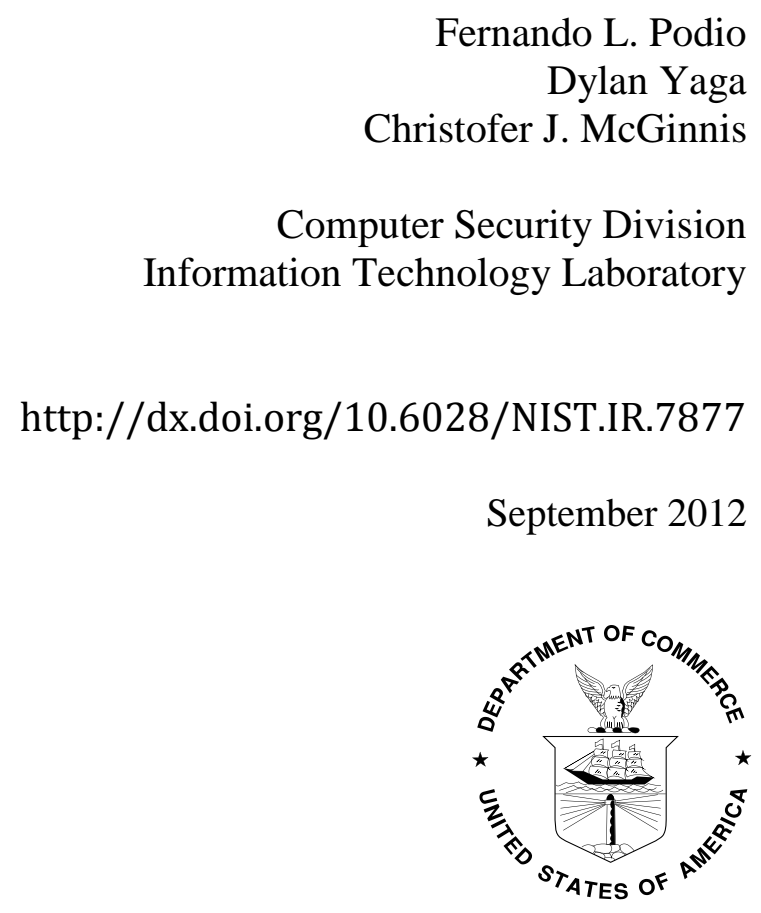

U.S. Department of Commerce Acting Secretary Rebecca M. Blank

National Institute of Standards and Technology Patrick Gallagher, Under Secretary for Standards and Technology and Director 
This page left intentionally blank 


\section{Reports on Computer Systems Technology}

The Information Technology Laboratory (ITL) at the National Institute of Standards and Technology (NIST) promotes the U.S. economy and public welfare by providing technical leadership for the Nation's measurement and standards infrastructure. ITL develops tests, test methods, reference data, proof of concept implementations, and technical analysis to advance the development and productive use of information technology. ITL's responsibilities include the development of technical, physical, administrative, and management standards and guidelines for the cost-effective security and privacy of sensitive unclassified information in Federal computer systems. This Interagency Report discusses ITL's research, guidance, and outreach efforts in computer security, and its collaborative activities with industry, government, and academic organizations.

\section{National Institute of Standards and Technology Interagency Report 39 pages (2012)}

Certain commercial entities, equipment, or materials may be identified in this document in order to describe an experimental procedure or concept adequately. Such identification is not intended to imply recommendation or endorsement by the National Institute of Standards and Technology, nor

is it intended to imply that the entities, materials, or equipment are necessarily the best available for the purpose. 


\begin{abstract}
The Computer Security Division of the Information Technology Laboratory at the National Institute of Standards and Technology (NIST/ITL) supports the development of biometric conformance testing methodology standards and other conformity assessment efforts through active technical participation in the development of these standards and the associated conformance test architectures and test suites. BioCTS 2012 is biometric conformance test software designed to test implementations for conformance to various biometric data interchange format standards. BioCTS 2012 for the American National Standards Institute (ANSI)/NIST-ITL 1-2011 tests implementations of NIST SP 500-290 ANSI/NIST ITL 1-2011 (AN-2011) "Data Format for the Interchange of Fingerprint, Facial \& Other Biometric Information" using test assertions documented in NIST SP 500-295, "Conformance Testing Methodology for ANSI/NIST-ITL 1-2011, Data Format for the Interchange of Fingerprint, Facial \& Other Biometric Information (Release 1.0)". BioCTS 2012 for the International Standards Organization/International Electrotechnical Commission (ISO/IEC) tests implementations of biometric data interchange formats developed by Subcommittee 37 - Biometrics of the Joint Technical Committee 1 - Information Technology of ISO and IEC. Support for testing Biometric Information Records (BIRs) conforming to instantiations of the Common Biometric Exchange Formats Framework (CBEFF) specified in national and international standards is also provided. BioCTS 2012 for ANSI/NIST-ITL 1-2011 is currently designed to support testing of implementations that include any of the Record Types defined in AN-2011, but conformance testing is only performed for the selected Record Types $(1,4,10,13,14,15$, and 17). Plans exist to extend the test tool to support additional Record Types. Information regarding BioCTS 2012 testing architectures, code structure, and other software design details is provided.
\end{abstract}

\title{
Disclaimer
}

Statements made in this paper should not be interpreted as standards, guidelines, best practices, or recommendations for specific changes to any other NIST publications.

\section{Feedback Suggested}

These Conformance Test Architectures and Test Suites, User Guides, and sample ("pass/fail”) data are available from the following web site: http://www.nist.gov/itl/csd/biometrics/biocta_download.cfm.

Feedback on BioCTS 2012, the sample data, and documentation is welcome. Please send comments to biocts@nist.gov.

\section{Acknowledgements}

The work discussed in this publication was sponsored, in part, by the Department of Homeland Security/ United States Visitor and Immigrant Status Indicator Technology (US-VISIT) Program. 


\section{Table of Contents}

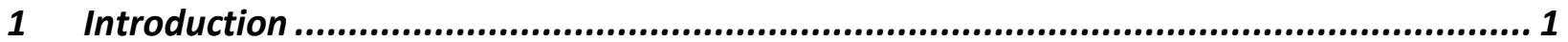

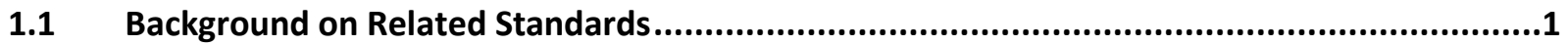

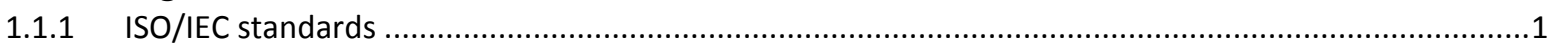

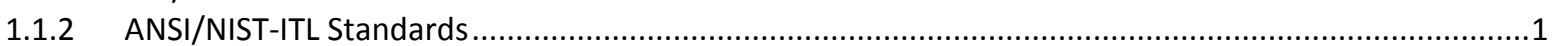

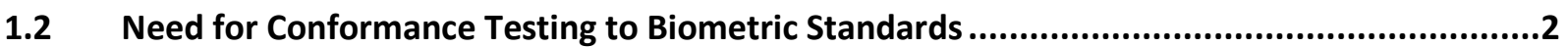

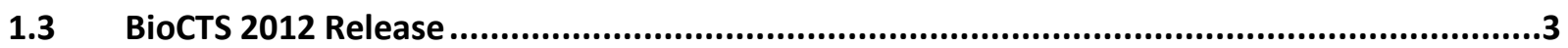

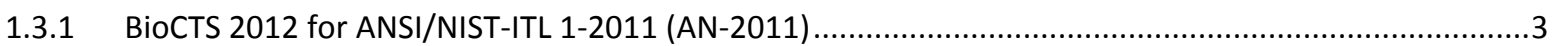

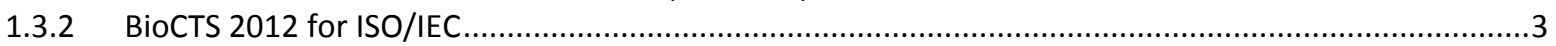

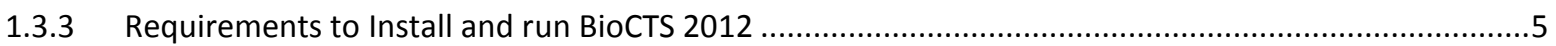

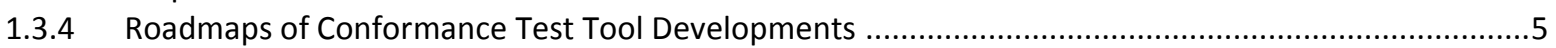

2 Standard Data Structures and BioCTS Design Philosophy .....................................6

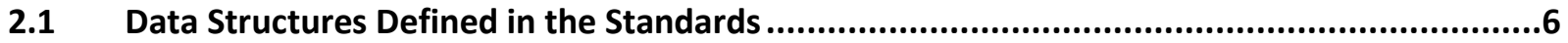

2.1.1 ISO/IEC 19794-x (2G) Biometric Data Interchange Formats .............................................................

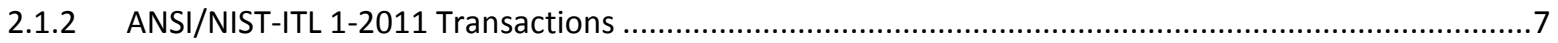

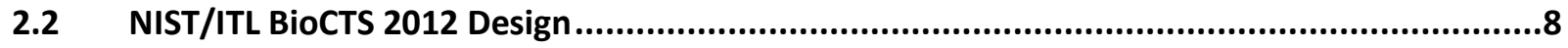

2.3 BioCTS 2012 for ISO/IEC standards and CBEFF BIRs ........................................................11

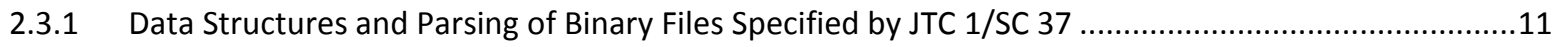

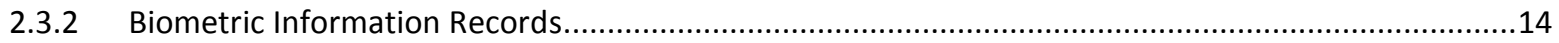

2.4 BioCTS 2012 for AN-2011 - Testing of AN-2011 High-Level Architecture ............................16

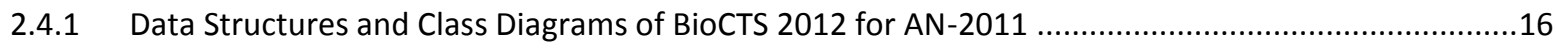

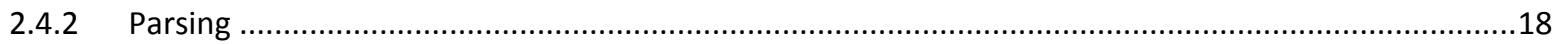

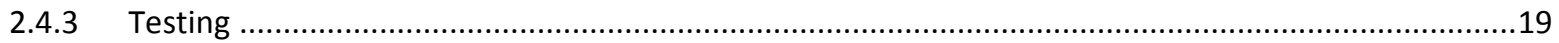

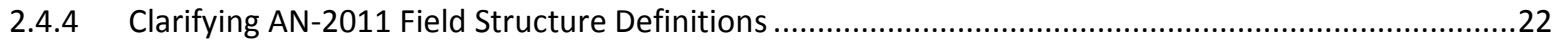

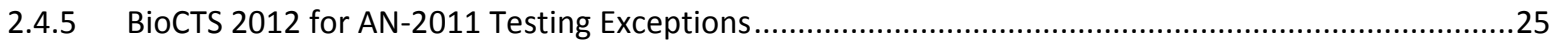

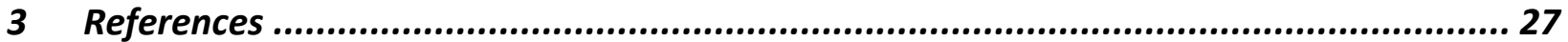

Annex A - Roadmaps of Biometric Data Interchange Formats and Conformance Testing Methodologies ................................................................................................ 28

A.1 First Generation of ISO/IEC Data Interchange Format Standards ..........................................28

A.2 Second Generation of ISO/IEC Data Interchange Format Standards .......................................29

Annex B - Roadmaps of Conformance Test Tools for Biometric Data Interchange Formats ... 30

B.1 CTSs Designed to Test Implementations of AN-2011 Transactions .........................................30

B.2 CTSs Designed to Test Implementations of ISO/IEC Data Interchange Formats .........................31

B.3 CTS Designed to Test Implementations of PIV profiles specified in NIST SP 800-76-2 ................32

\section{List of Figures and Tables}

Figure 2-1: Structure of JTC 1/SC 37 Biometric Data Interchange Records (BDIRs) ............................................... 7

Figure 2-2: High-level Structure of an ANSI/NIST-ITL 1-2011 Transaction..........................................................

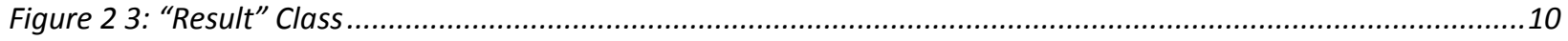

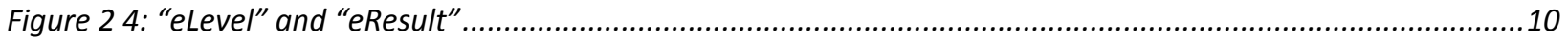




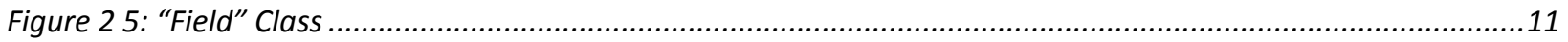

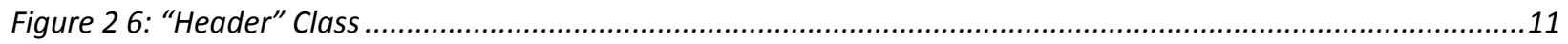

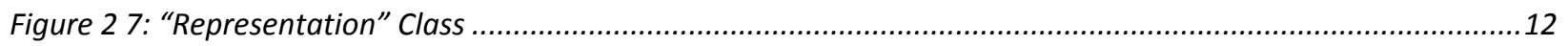

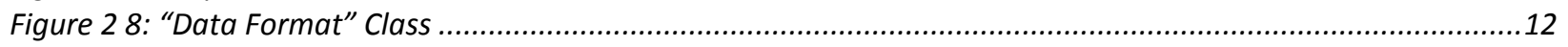

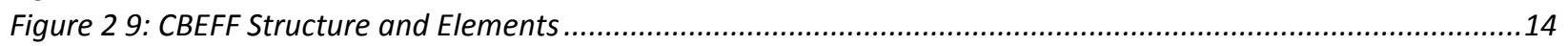

Figure 2 10: "BIR, PatronFormat", "DataFormat", and "SecurityFormat" Classes ..................................................15

Figure 2-11: BioCTS 2012 for ISO/IEC High-Level Architecture .......................................................................16

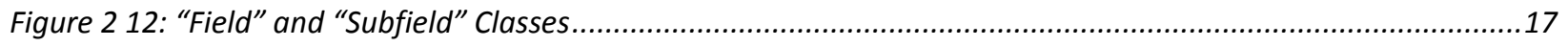

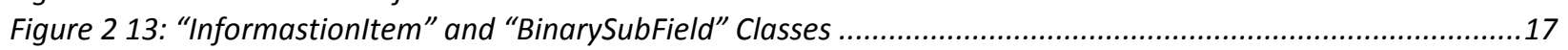

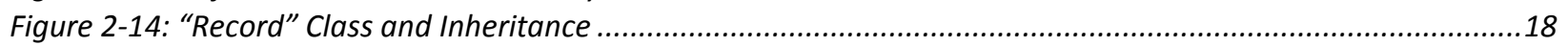

Figure 2 15: BioCTS 2012 for AN-2011 High-Level Architecture .............................................................................21

Table 2-1: Comparison of the Field Structure in the Standard and the Code ......................................................24.

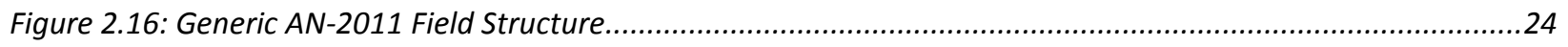

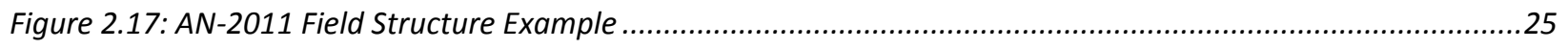

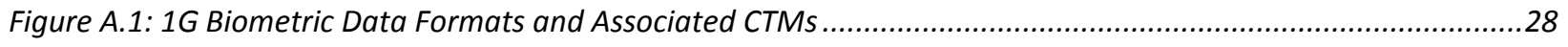

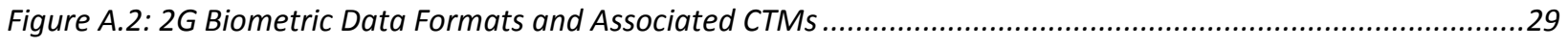

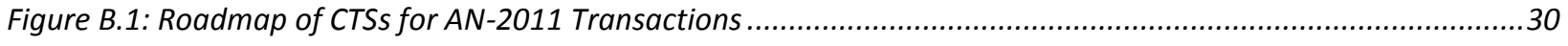

Figure B.2: Roadmap of CTSs for ISO/IEC Data Interchange Formats ...........................................................

Figure B.3: Roadmap of CTSs for PIV Profiles Defined in NIST SP 800-76-2 ...................................................32 


\section{Introduction}

\subsection{Background on Related Standards}

The success of biometric applications is particularly dependent on the interoperability of biometric systems. Deploying these systems requires a comprehensive portfolio of biometric standards developed in support of interoperability and data interchange.

\subsubsection{ISO/IEC standards}

The international standards development body responsible for developing a comprehensive portfolio of biometric standards is ISO/IEC JTC 1/SC 37 - Biometrics, a Subcommittee of the Joint Technical Committee 1 of the International Standards Organization/International Electrotechnical Commission (ISO/IEC) [1]. The Subcommittee operates under the principles of international standards development based on three key ISO principles: consensus, industry wide representation, and voluntary standards. Within its portfolio of development projects, JTC 1/SC 37's scope of work includes the development of biometric data interchange formats for a number of biometric modalities and their associated conformance testing methodology standards. Figure A.1 depicts the roadmap of the "first generation" (1G) of biometric data interchange formats and associated conformance testing methodologies developed or under development in this Subcommittee. The $1 \mathrm{G}$ biometric data interchange formats are specified in binary encoding. As shown in the roadmap, these data formats and most of the associated conformance testing methodologies are published (as indicated by the publication year).

Figure A.2 depicts the "second generation" (2G) of biometric data interchange formats and associated conformance testing methodologies. The $2 \mathrm{G}$ data interchange formats (most of them have been published) are specified in binary encoding. As shown in the roadmap, amendments to most of the $2 \mathrm{G}$ standards are being developed to specify the equivalent Extensible Markup Language (XML) encoding formats. The $2 \mathrm{G}$ conformance testing methodologies target the binary encoding formats. The Subcommittee recently initiated the development of the equivalent testing methodologies for the XML encoding formats specified in the $2 \mathrm{G}$ data interchange formats.

\subsubsection{ANSI/NIST-ITL Standards}

The American National Standards Institute/National Institute of Standards and TechnologyInformation Technology Laboratory (ANSI/NIST-ITL) standard "Data Format for the Interchange of Fingerprint, Facial \& Other Biometric Information" is used by law enforcement, intelligence, military, and homeland security organizations throughout the world. The first version of the standard dates to 1986. Over the years, it has been updated and expanded to cover more biometric modalities beyond the original record type of fingerprint minutiae. ANSI/NIST-ITL 1-2011, NIST Special Publication 500-290 "Data Format for the Interchange of Fingerprint, Facial \& Other Biometric Information" (AN-2011) supersedes all previous versions and amendments to the standard [2].

AN-2011 specifies two data encoding formats: Traditional format and National Information Exchange Model (NIEM)-conformant XML format. New modalities (DNA and plantars) were added as new record types. The extended feature set was added to Record Type 9; Record Type 10 was extended to include all body part images and to include anthropomorphic image markups; and compact iris image storage formats were introduced. There were substantial metadata upgrades as 
well, including geographic location, data handling logs, original source and associated reference data. Information assurance capabilities were added as Record Type-98.

An initial version of an associated conformance testing methodology (CTM) for AN-2011 was developed by the NIST/ITL CTM Working Group. This Working Group was established by NIST/ITL and is chaired by NIST/ITL staff. The group is made up of NIST experts as well as experts from other US Government agencies and industry. The initial release of this document is NIST Special Publication 500-295, "Conformance Testing Methodology for ANSI/NIST-ITL 1-2011, Data Format for the Interchange of Fingerprint, Facial \& Other Biometric Information (Release 1.0)" [3]. NIST SP 500-295 specifies over twelve hundred test assertions for the following sections and Record Types of the AN-2011 standard:

- Section 5: Data Conventions

- Section 7: Information Associated with Several Record Types

- Section 8.1 Record Type-1: Transaction information record

- Section 8.4 Record Type-4: Grayscale fingerprint image

- Section 8.10 Record Type-10: Facial, other body part and Scar, Mark, and Tattoo (SMT) image record

- Section 8.13 Record Type-13: Friction-ridge latent image record

- Section 8.14 Record Type-14: Fingerprint image record

- Section 8.15 Record Type-15: Palm print image record

- Section 8.17 Record Type-17: Iris image record

- Annex B: Traditional Encoding

\subsection{Need for Conformance Testing to Biometric Standards}

The existence of biometric standards alone is not enough to demonstrate that products meet the technical requirements specified in the standards. Conformance testing captures the technical description of a specification and measures whether an implementation faithfully implements the specification. Conformance testing provides developers, users, and purchasers with increased levels of confidence in product quality and increases the probability of successful interoperability.

Although no conformance test can be comprehensive enough to test all the different combinations of mandatory requirements of a standard and all possible combinations of conditional and optional characteristics that could be included in the standards, a well-designed conformance test tool that faithfully implements a standard conformance testing methodology could raise the level of confidence on the test results. Therefore, a set of implementations tested with such a tool (and reported to be conformant to the standard), will be more likely to conform to the standard.

The Computer Security Division (CSD) of NIST/ITL supports the development of biometric conformance testing methodology standards and other conformity assessment efforts through active technical participation in the development of biometric standards and associated conformance test architectures and test suites. NIST/ITL CSD develops these test tools to support users that require conformance to selected biometric standards and product developers interested in conforming to biometric standards by using the same testing tools available to users. Testing laboratories can also benefit from the use of these test tools. These efforts support the possible establishment of conformity assessment programs to validate conformance to biometric standards. 


\subsection{BioCTS 2012 Release}

As shown below, BioCTS 2012 includes two architectures:

- BioCTS 2012 for AN-2011

- BioCTS 2012 for ISO/IEC

The BioCTS 2012 software was released in August 2012. Installer versions of these test tools, sample data ("pass"/"fail" implementations), and associated documents are available for download at: http://www.nist.gov/itl/csd/biometrics/biocta_download.cfm.

The release includes the Installer versions of the test tools listed in Sec. 1.3.1 to Sec. 1.3.3.

\subsubsection{BioCTS 2012 for ANSI/NIST-ITL 1-2011 (AN-2011)}

BioCTS 2012 for AN-2011 is a Conformance test architecture and test suite designed to test AN2011 transactions. Conformance testing is performed for the test assertions documented in NIST SP 500-295, which specifies over twelve hundred test assertions for the following sections and Record Types of the AN-2011 standard:

- Section 5: Data Conventions

- Section 7: Information Associated with Several Record Types

- Section 8.1 Record Type-1: Transaction information record

- Section 8.4 Record Type-4: Grayscale fingerprint image

- Section 8.10 Record Type-10: Facial, other body part and Scar, Mark, and Tattoo (SMT) image record

- Section 8.13 Record Type-13: Friction-ridge latent image record

- Section 8.14 Record Type-14: Fingerprint image record

- Section 8.15 Record Type-15: Palm print image record

- Section 8.17 Record Type-17: Iris image record

- Annex B: Traditional Encoding

For transactions that include record types other than those listed above, the presence of the records is reported (including record length), but no conformance testing is performed.

\subsubsection{BioCTS 2012 for ISO/IEC}

BioCTS for ISO/IEC is a Conformance Test Architecture (CTA) that supports Conformance Test Suites (CTSs) designed to test implementations of ISO/IEC biometric data interchange format standards developed by JTC 1/SC 37 - Biometrics and selected PIV profiles (iris data format). The toolset includes CTSs designed to test implementations of:

- ISO/IEC Biometric data interchange formats

- Common Biometric Exchange Formats Framework (CBEFF) Biometric Information Records (BIR)

- Individual components of the CBEFF BIR

The following is a list of the available test tools and the relevant standards used in their development.

First Generation (1G) of ISO/IEC Data Interchange Formats

- ISO/IEC 19794-2: 2005 
○ ISO/IEC 19794-2:2005, Biometric data interchange formats - Part 2: Finger minutiae data

○ ISO/IEC 19794-2:2005 TECHNICAL CORRIGENDUM 1

○ ISO/IEC 29109-2:2010 Conformance testing methodology for biometric data interchange formats defined in ISO/IEC 19794 -- Part 2: Finger minutiae data

- ISO/IEC 19794-4: 2005

○ ISO/IEC 19794-4:2005, Biometric data interchange formats -- Part 4: Finger image data

○ ISO/IEC 19794-4:2005 TECHNICAL CORRIGENDUM 1

- ISO/IEC 29109-4:2010, Conformance testing methodology for biometric data interchange formats defined in ISO/IEC 19794 -- Part 4: Finger image data

○ ISO/IEC 29109-4:2010 TECHNICAL CORRIGENDUM 1

- ISO/IEC 19794-5: 2005

○ ISO/IEC 19794-5:2005, Biometric data interchange formats -- Part 5: Face image data

○ ISO/IEC 19794-5:2005 TECHNICAL CORRIGENDUM 1

○ ISO/IEC 19794-5:2005 TECHNICAL CORRIGENDUM 2

- ISO/IEC 29109-5:2012, Conformance testing methodology for biometric data interchange formats defined in ISO/IEC 19794 -- Part 5: Face image data

\section{Second Generation (2G) of ISO/IEC Data Interchange Formats}

- ISO/IEC 19794-2: 2011

○ ISO/IEC 19794-2:2011, Biometric data interchange formats -- Part 2: Finger minutiae data

- ISO/IEC 19794-2:2011 EDITORIAL CORRIGENDUM 1

- ISO/IEC JTC 1/SC 37 N 4902 Text of 19794-2 3rd PDAM 1, Biometric data interchange formats - Part 2: Finger minutia data - Amendment 1: Conformance testing methodology

- ISO/IEC 19794-4: 2011

○ ISO/IEC 19794-4:2011, Biometric data interchange formats -- Part 4: Finger image data

- ISO/IEC 19794-4:2011 EDITORIAL CORRIGENDUM 1

- ISO/IEC JTC 1/SC 37 N 4944 Text of 19794-4 DAM1, Biometric data interchange formats: Part4: Finger image data - Amendment1: Conformance testing methodology

- ISO/IEC 19794-6: 2011

○ ISO/IEC 19794-6:2011, Biometric data interchange formats -- Part 6: Iris image data

○ ISO/IEC 19794-6:2011 EDITORIAL CORRIGENDUM 1

- ISO/IEC JTC 1/SC 37 N 4881 Text of 19794-6 3rd PDAM 1, Biometric data interchange formats - Part 6: Iris image data - Amendment 1: Conformance testing methodology

\section{PIV Profile of ISO/IEC 19794-6:2011 (specified in NIST SP 800-76-2) ${ }^{1}$}

- CBEFF Header of the PIV 19794-6:2011 Profile

○ As defined in NIST SP 800-76-2 (Draft) Table 15 "Patron format PIV specification"

- PIV Profile of ISO/IEC 19794-6: 2011 On-Card

\footnotetext{
${ }^{1}$ Specified by the NIST SP 800-76-2 Patron Format PIV Specification, currently in draft form. BioCTS 2012 for ISO/IEC will be updated as NIST SP 800-76-2 progresses through final publication.
} 
○ In addition to the ISO/IEC 19794-6:2011 standard this CTS implements requirements specified in NIST SP 800-76-2 (Draft) "Table 11 - ISO/IEC 19794-6 profile for iris images stored on PIV Cards"

- PIV Profile of ISO/IEC 19794-6: 2011 Off-Card

○ In addition to the ISO/IEC 19794-6:2011 standard this CTS implements requirements specified in NIST SP 800-76-2 (Draft) "Table 12 - ISO/IEC 19794-6 profile for iris images stored outside PIV Cards"

- CBEFF Opaque Security Block

- The Opaque SB CTS accepts any data passed to it. No standard was used to implement this. No conformance testing is performed.

The CTSs released under CTA Beta Implementation 2.0 in September of 2011 have been migrated to BioCTS 2012 and are no longer available. Those included CTSs designed to test implementations of selected $1 \mathrm{G}$ of ISO/IEC data interchange formats, 2G iris data interchange format 19794-6:2011and the PIV profiles of the ISO/IEC 19794-6:2011 standard,

A roadmap of test tool development depicting the test tools available as well as the tools under development or planned is included in Annex A.

\subsubsection{Requirements to Install and run BioCTS 2012}

Both architectures of BioCTS 2012 and the associated conformance test suites are supported by several Microsoft@ Operating Systems:

- Windows ${ }^{\circledR}$ XP ${ }^{\mathrm{TM}}$ Service Pack 3

- Windows ${ }^{\circledR}$ Vista ${ }^{\mathrm{TM}}$ Service Pack 2

- Windows ${ }^{\circledR}{ }^{\mathrm{TM}}$ Service Pack 1

BioCTS 2012 requires Microsoft ${ }^{\circledR}$.NET 4.0 Framework, which can be downloaded from the Microsoft@ Download Center [4].

\subsubsection{Roadmaps of Conformance Test Tool Developments}

Annex B discusses roadmaps of the development of conformance test tool designed to test implementations of a number of biometric data interchange formats. In addition to identifying the existing tools included in BioCTS 2012 for both ISO/IEC standards and ANSI/NIST-ITL 1-2011, plans for further tool developments are addressed. The plans for further development include a revised version(s) of BioCTS 2012 for AN-2011 that provides tests for additional Record Types (e.g., DNA, Dental, Voice) and the NIEM-XML encoding; additional biometric data interchange formats developed by ISO/IEC Joint Technical Committee 1 Subcommittee 37 (JTC 1/SC 37) for both binary encoding and XML encoding; and test tools for additional PIV profiles of biometric data interchange standards specified in NIST Special Publication 800-76-2 (currently a draft publication) [5]. These roadmaps are depicted in Fig. B.1 to B.3. 


\section{Standard Data Structures and BioCTS Design Philosophy}

\subsection{Data Structures Defined in the Standards}

Sections 2.1.1 and 2.1.2 addresses the differences between the second generation of biometric data interchange formats developed by JTC 1/SC 37 (2G) and the ANSI/NIST-ITL 1-2011 Transactions. ISO/IEC 19794 "Information technology — Biometric data interchange formats" is one of a family of international standards being developed by ISO/IEC JTC 1/SC 37 that support interoperability and data interchange among biometric applications and systems. The data interchange formats and containing structures found in JTC 1/SC 37 Biometric Data Interchange Records and ANSI/NISTITL 1-2011 Transactions are outlined below.

\subsubsection{ISO/IEC 19794-x (2G) Biometric Data Interchange Formats}

Figure 2-1 shows the Biometric Data Interchange Record (BDIR) defined in the ISO/IEC 19794-X standards. The data structure of the second generation of biometric data interchange formats is comprised of:

- Fields containing Data;

- A General Header containing Fields; and

- One or more Representations containing Fields.

The general header and the first representation are mandatory in the data format for each biometric modality. Additional representation data structures are optional (may be contained in the record or not). The General Header contains fields such as the format identifier, the version number, and the number of representations contained in the record. Representations are comprised of a representation header (distinct for each record format). The representation header contains mandatory fields such as capture date and time of the biometric sample contained in the representation and optional fields such the capture device identifier and quality field. 


\section{General Header}

- Field 1

- Field 2

- Field 3

- $\quad$........

$\bullet$

Representation 1

- Field 1

- Field 2

- Field 3

- $\quad$.........

-

\section{Representation 2}

- Field 1

- Field 2

- Field 3

- $\quad \cdots . . . . .$.

Figure 2-1: Structure of JTC 1/SC 37 Biometric Data Interchange Records (BDIRs)

\subsubsection{ANSI/NIST-ITL 1-2011 Transactions}

Figure 2-2 below depicts the ANSI/NIST-ITL 1-2011 Transaction structures, which are comprised of:

- Transactions which contain Records

- Records which contain Fields

- Fields which may contain Subfields, Information Items, or data

- Subfields which may contain one or more Information Items or data

- Information Items which contain data 


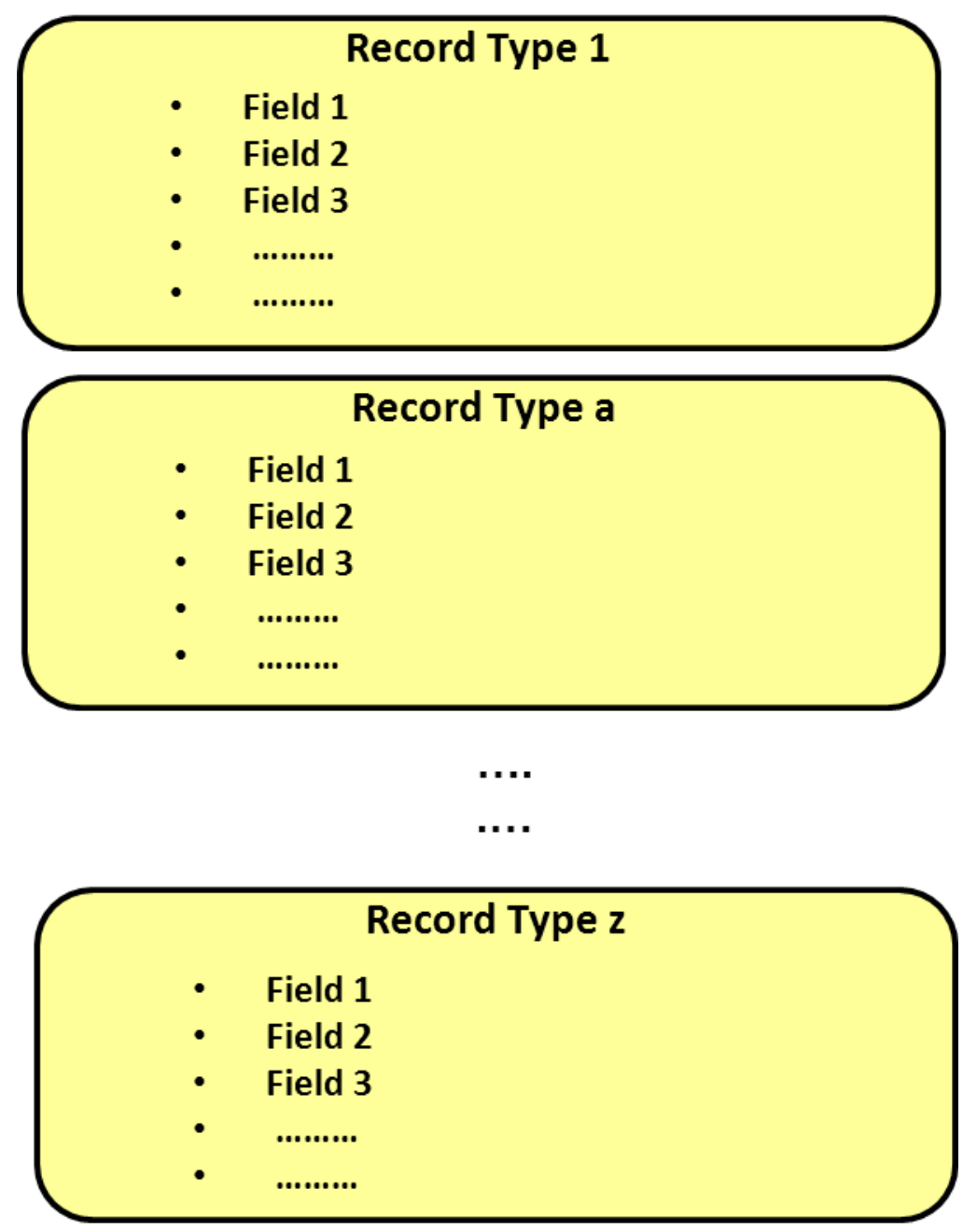

Figure 2-2: High-level Structure of an ANSI/NIST-ITL 1-2011 Transaction

Figure 2.16 in Sec. 2.4.4 depicts the generic AN-2011 Field Structure.

Record Type- 1 is required and shall always be the first record within the transaction. In addition to Record Type-1, the standard requires that there shall be at least one other record type included in the transaction. There may be multiple records in a transaction of each record type other than Type-1. A record is comprised of fields. Each field is assigned a number, a description, and a mnemonic (e.g., Field 10.020: Subject pose / POS).

\subsection{NIST/ITL BioCTS 2012 Design}

The software developed for both architectures (BioCTS 2012 for ISO/IEC and BioCTS 2012 for AN2011) was developed in C\# using the Microsoft ${ }^{\circledR}$.NET 4.0 Framework. The programming paradigm chosen was Object-oriented programming (OOP) which uses objects (data structures consisting of data fields and methods together with their interactions) that are instances of classes (which contain definitions for creating instances of objects - referred to as class instances, class objects, instance objects or simply objects). A class defines constituent members which enable its instances (objects) to have state and behavior. Classes were used to define each component in the BioCTS architecture. 
Despite differences in their structure, the major design philosophy for both Conformance Test Architectures and their Conformance Test Suite(s) is the use of encapsulation and polymorphism. Generally, encapsulation is a term used to describe how access to objects' data is restricted, and polymorphism allows different data types and objects to be treated uniformly in code using interfaces. Encapsulation was used in NIST/ITL BioCTS 2012 to bundle data with its testing methods and to restrict access to other components of the same level as much as possible. Since the components do not know about other components at the same level, they do not rely on them to function properly.

- A Field contains

o Name

- Data

- Tests to Perform

- Results of Tests

- A Record or Header (AN-2011)/Representation (JTC 1/SC 37) contains

- Name

- Fields it contains

- Tests to Perform

○ Results of Tests

- A Transaction (AN-2011)/BDIR (JTC 1/SC 37) contains

- Name

○ Records or Header/Representations it contains

○ Tests to Perform

- Results of Tests

Given two fields, $F_{1}$ and $F_{2}$ contained in the same record, $F_{1}$ cannot access any of the contents of $F_{2}$ and vice versa; however, the record that contains them can access the contents of both fields. The same can be said of two records contained within the same transaction. Neither record can access the contents of the other, but the transaction that contains the records can access the contents of both. This independence among components allows immediate testing of the code rather than waiting on dependent modules to be completed.

Reference [3] "NIST Special Publication 500-295, Conformance Testing Methodology for ANSI/NIST-ITL 1-2011, Data Format for the Interchange of Fingerprint, Facial \& Other Biometric Information" defines three levels of conformance testing: Level 1 (L1), Level 2 (L2) and Level 3 (L3). These three levels apply to both BioCTS 2012 for AN-2011 as well as BioCTS 2012 for ISO/IEC standards.

The current version of BioCTS does not perform Level 3 conformance tests but it is designed to incorporate this level of testing at a later time. Every test in BioCTS 2012 returns a "Result", which contains a Test Level, Test Message, Test Name, and an "eResult". Figure 2-3 depicts the "Result" class. Some enumerated types such as "eLevel" and "eResult" were created to standardize these values throughout BioCTS. Parse-level tests defined by the "eLevel" enumerated type shown in Fig. 2-4 are performed before all other levels of testing. In addition to "Pass"/"Fail", the test results provide "eResult" values ranging from "Ok" to "Critical Error". 


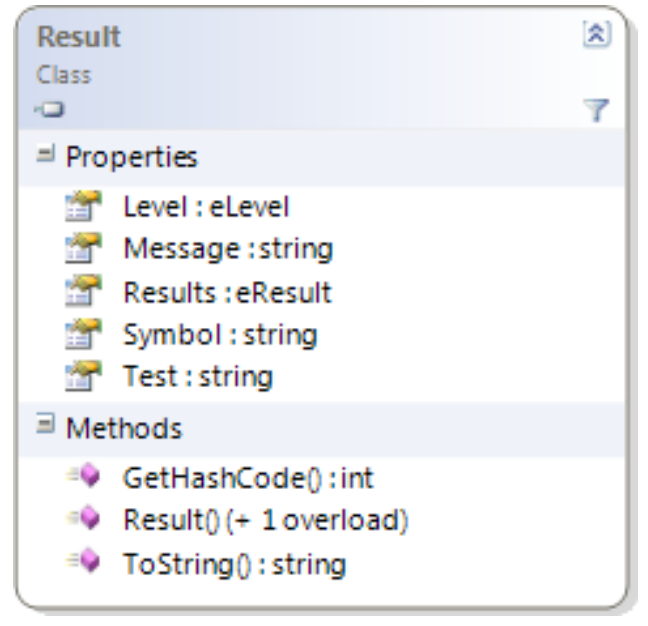

Figure 2 3: "Result" Class

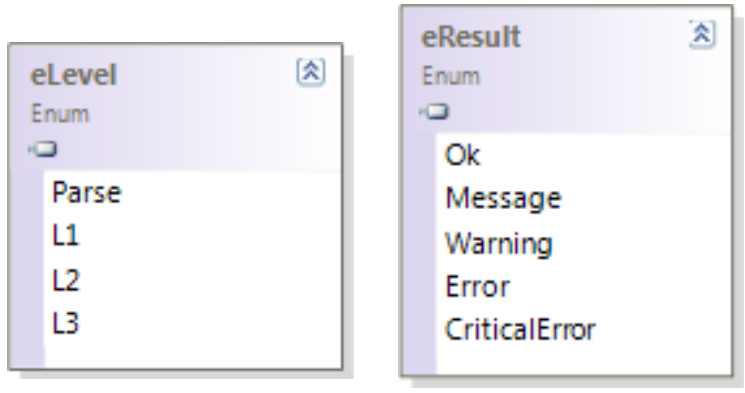

Figure 2 4: "eLevel" and "eResult" 


\subsection{BioCTS 2012 for ISO/IEC standards and CBEFF BIRs}

\subsubsection{Data Structures and Parsing of Binary Files Specified by JTC 1/SC 37}

The ISO/IEC 19794-x series of "1G" standards specifies binary encoding only. The Second Generation of these standards specifies both a binary format and an XML format in standards amendments.

Unlike parsing binary files, parsing XML is well-defined and streamlined. Without set markers, or tags, parsing of binary format files relies on field length as defined in the standards. Thus, optional and variable-length fields present challenges during parsing.

BioCTS 2012 for ISO/IEC defines each Field as an individual class derived from the base Field class shown in Fig. 2-5. Each field inherits from this base class, and is self-contained.

For example, the "FormatIdentifier Field" Class is derived from the base Field class, but assigns values to the following:

- Its name is "Format Identifier"

- It is Field Number 1

- It has a Minimum Length of 4 bytes

- It has a Maximum Length of 4 bytes

- What Data (bytes) were parsed to it

- A list of Results

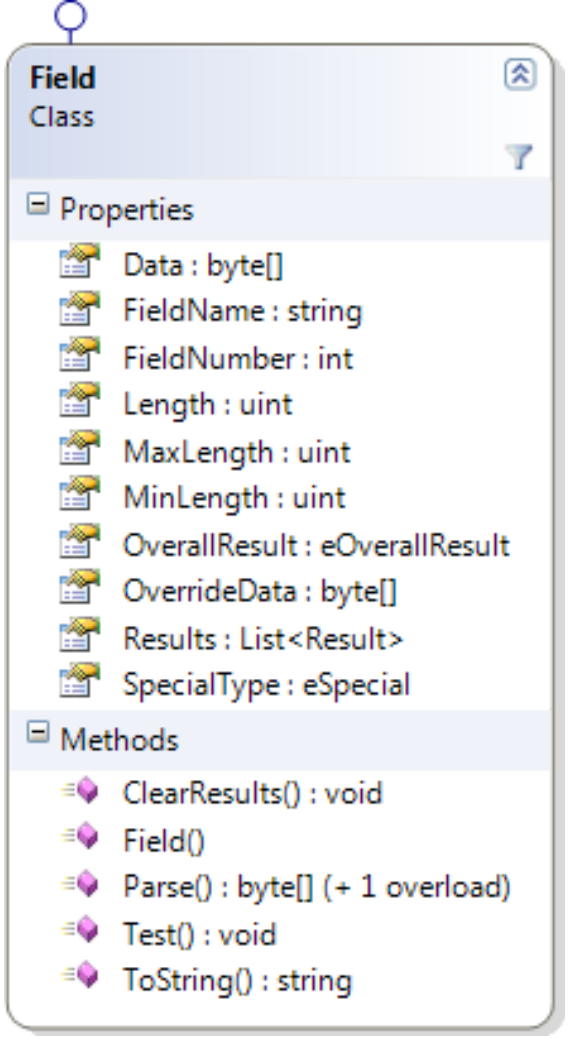

Figure 2 5: "Field" Class

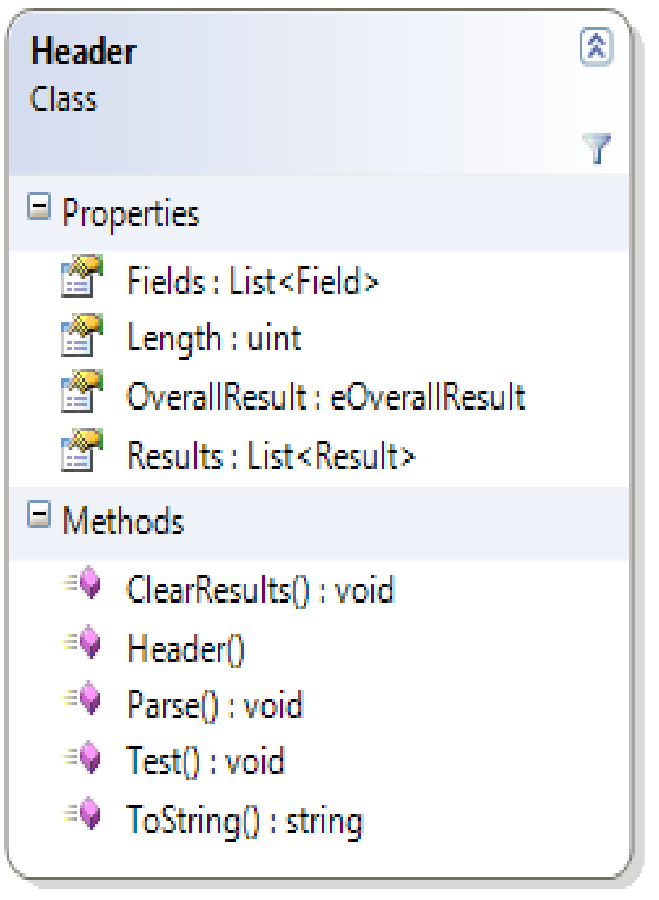

Figure 2 6: “Header” Class
However, FormatIdentifier Field does not have access to any of the other derived Fields. As was discussed in Sec. 2.1.1, Fields are the simplest building blocks of the ISO/IEC data interchange formats, which are then grouped together into sections to define General Headers or Representations. BioCTS 2012 for ISO/IEC has been designed to mimic the structure of the ISO/IEC standards when possible. For example, it has a Data Structure for the General Headers and Representations. Figure 2-6 depicts the Header class. A Header class has the ability to run tests as well; however, Level-2 tests that involve only the fields within a Header are rare.

The ISO/IEC biometric data interchange formats standards specify that the Biometric Information Data Record can contain multiple Views/Representations. A Representation has specific information about the biometric sample that was captured. It is made up of multiple Fields, collected in a List. 
The Representation class shown in Fig. 2-7, has the ability to perform Level-2 tests from its Test method and can perform any Level-2 test that requires only Fields from that Representation (e.g., comparing the Image's internal width/height vs. the representation's specified width/height).

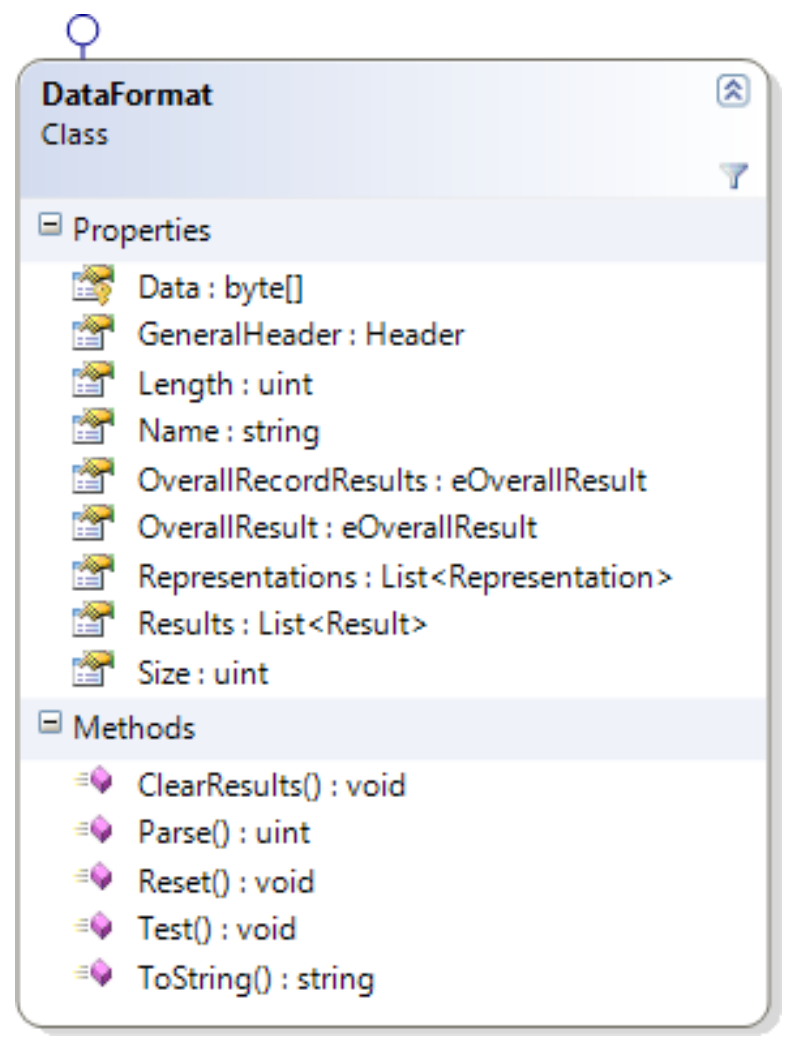

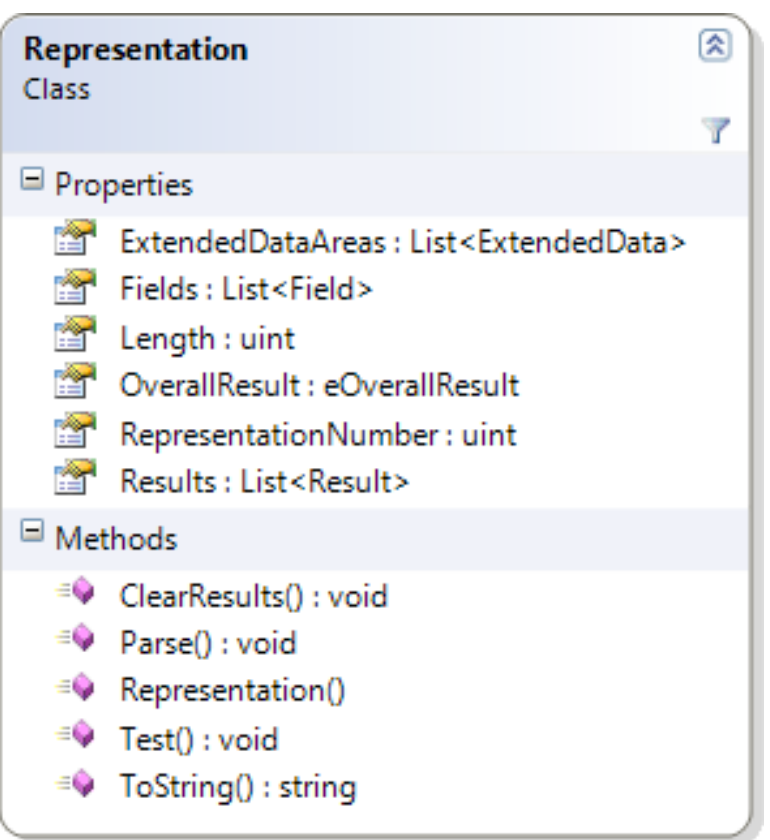

Figure 2 7: "Representation” Class

Some of the ISO/IEC standards specify that a Representation has Extended Data to accommodate annotations or vendor specific information. BioCTS 2012 has an optional area to parse Fields within an Extended Data area. Extended Data areas are very similar to Representations and Headers, in that they have a collection of Fields and a Test() method.

\section{Figure 2 8: "Data Format" Class}

The DataFormat class, depicted in Fig. 2-8, is the main class for a Conformance Test Suite (CTS). The ISO/IEC data interchange format standards specify that there is one Header and multiple Representations, and this is reflected in the DataFormat class. There is a single Header (GeneralHeader), and a List<Representation $>$ (list of Representations). Since the DataFormat class shown in Fig. 2-8 is the main class for a CTS, its Parse method is the first method called when testing an implementation, as shown in the pseudo code below.

When a DataFormat Parse() method is called, it parses the entire file incrementally. Pseudo code for "DataFormat Parse", "Header Parse" and "Representation" Methods are shown below.

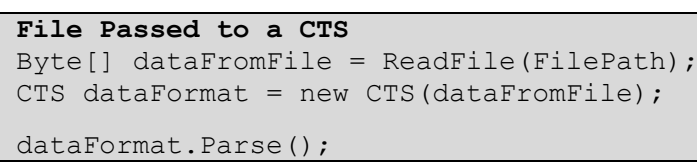




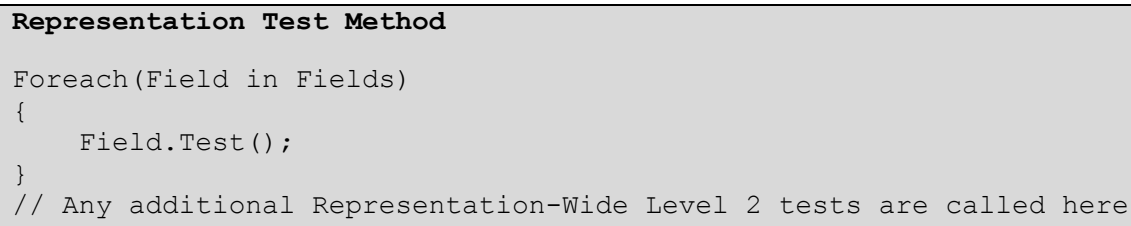

\subsubsection{Biometric Information Records}

Figure 2-9 depicts the CBEFF structure and its three elements: (a) a Standard Biometric Header (SBH); (b) the Biometric Data Block (BDB) which contains the biometric data (independently of the biometric modality); and (c) an optional Security Block (SB). This CBEFF structure is called a Biometric Information Record or BIR.

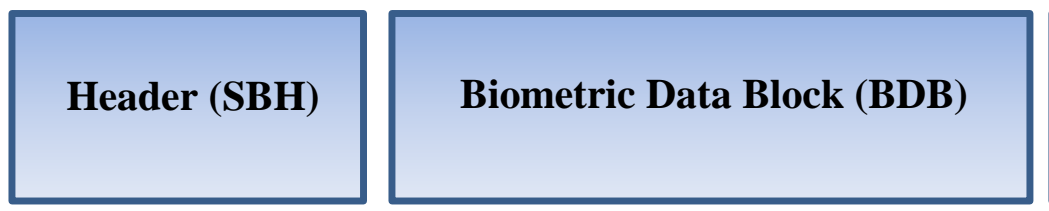

Security Block (SB)

Figure 2 9: CBEFF Structure and Elements

"BIR" is a superstructure within BioCTS 2012 that contains the following classes, shown in Fig. 210 :

- BIR class

- Patron Format class

- Data Format

- Security Format class 


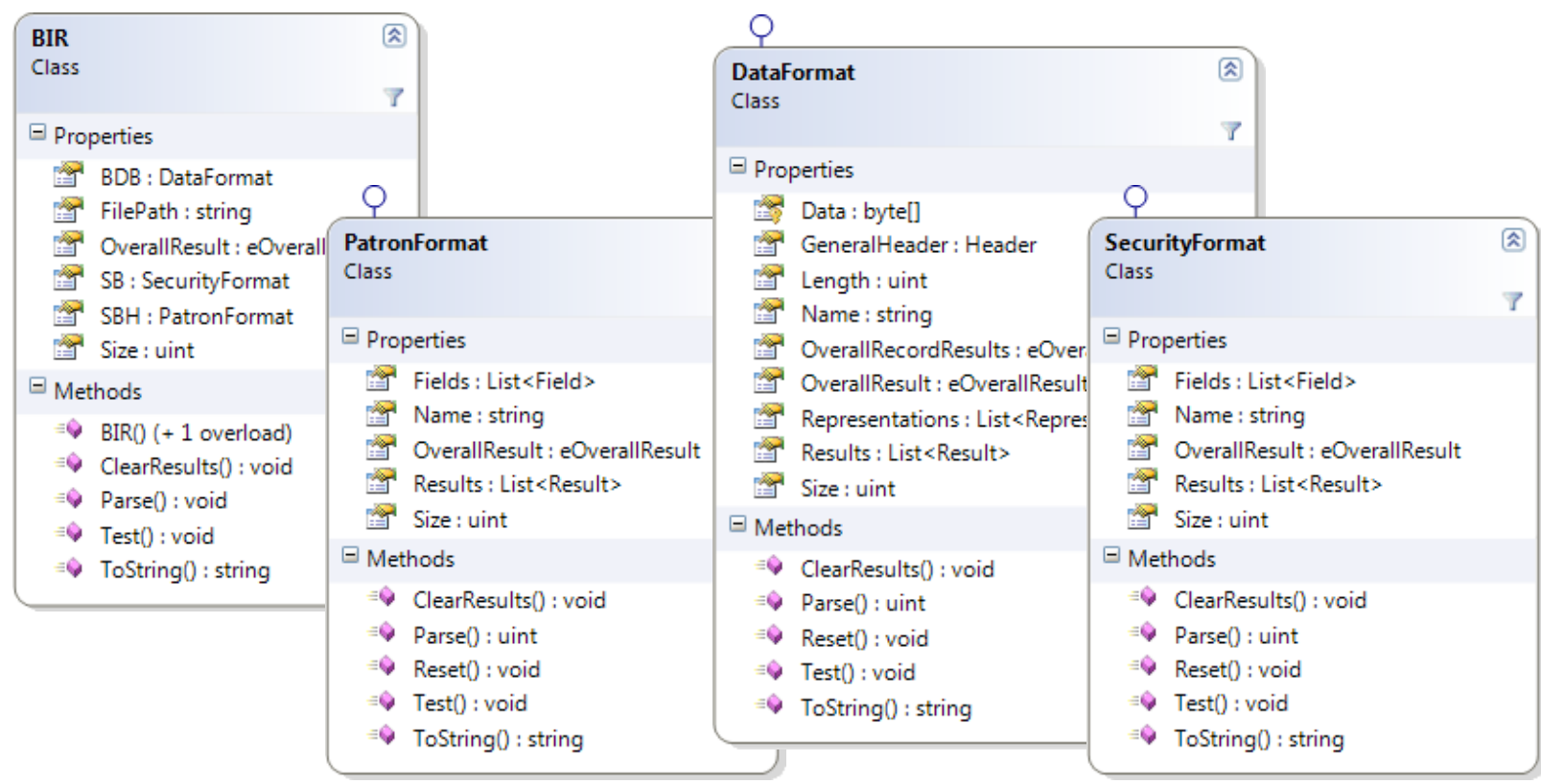

Figure 2 10: "BIR, PatronFormat”, “DataFormat”, and "SecurityFormat” Classes

BioCTS 2012 for ISO/IEC uses a modular architecture represented in Fig. 2-11 allowing the user to select each portion of the architecture. The software will automatically parse and test what is specified. 


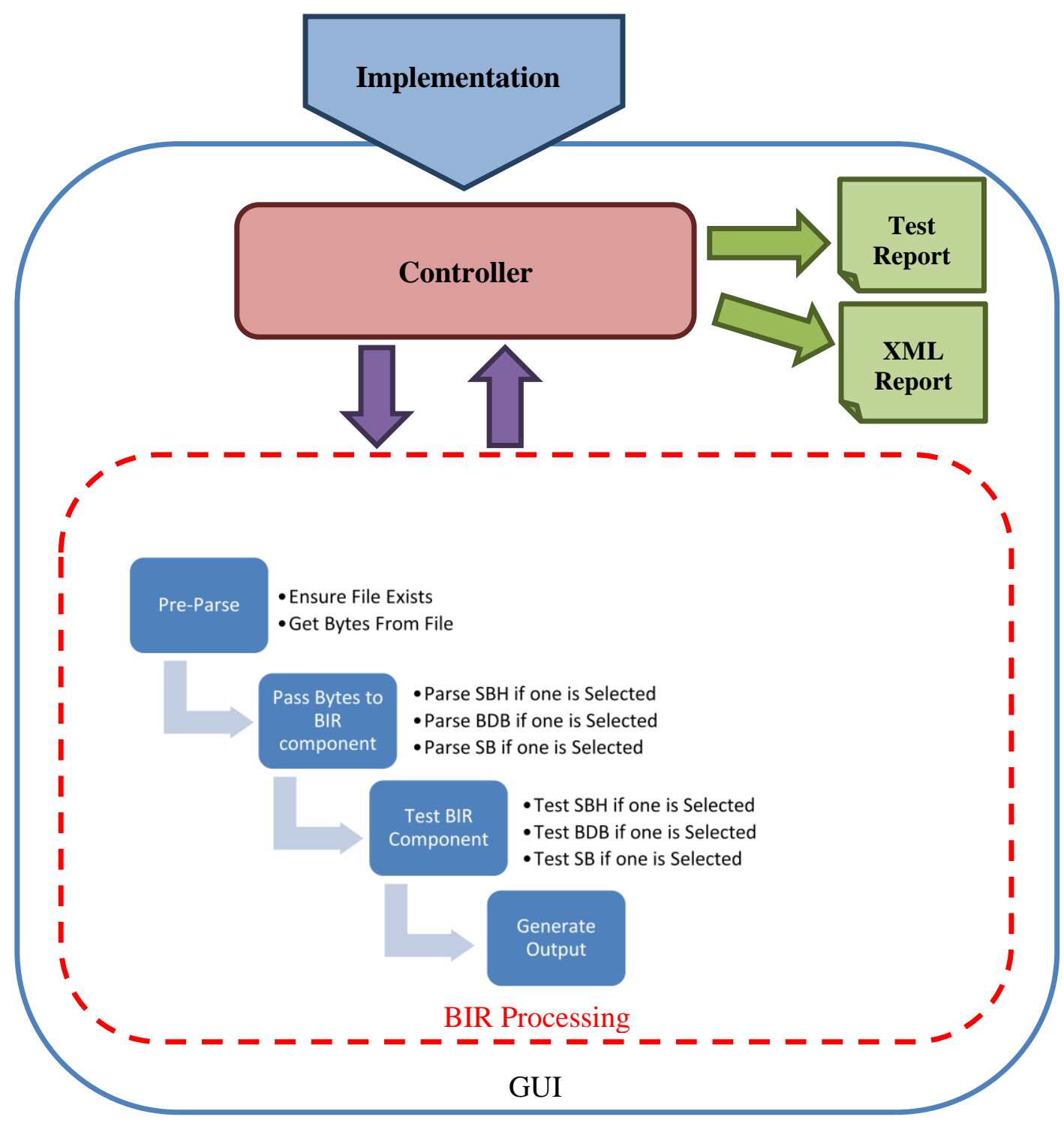

Figure 2-11: BioCTS 2012 for ISO/IEC High-Level Architecture

\subsection{BioCTS 2012 for AN-2011 - Testing of AN-2011 High-Level Architecture}

\subsubsection{Data Structures and Class Diagrams of BioCTS 2012 for AN-2011}

ANSI/NIST-ITL 1-2011 Transactions are more complex than the ISO/IEC data interchange formats because they include Records for a number of modalities, field structures made up of various combinations of subfields and information items, and a combination of binary and tagged Records. BioCTS 2012 for AN-2011 treats the Information Item as the smallest portion of Data of an AN2011 transaction. An Information Item may access only its own content and not that of any other structure. An Information Item has the ability to test itself, using its Test() method. "Field", "Subfield", "InformationItem", and "BinarySubfiled" classes used in BioCTS for AN-2011 are depicted in Fig. 2-12 and 2-13. Tests are generally performed on data contained in Information Items. A Subfield contains one or more Information Items, but does not contain data. Therefore, Subfields 
do not have the ability to perform tests. A Field is a collection of Subfields, and therefore, the Field class is able to test the Subfields held within it.

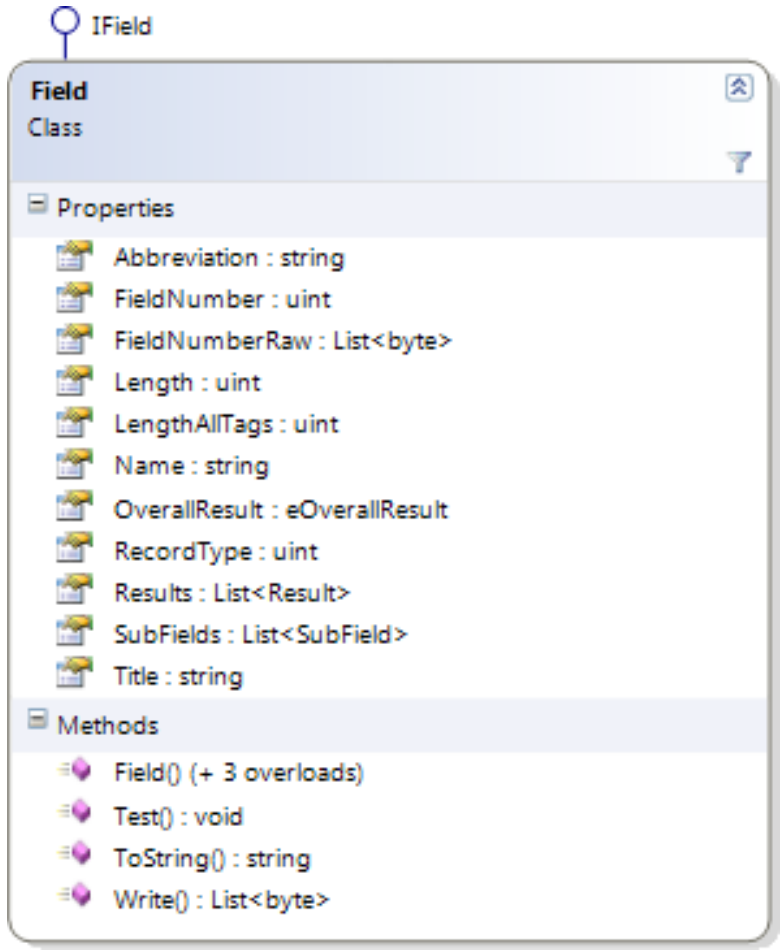

\begin{tabular}{|c|c|}
\hline $\begin{array}{l}\text { SubField } \\
\text { Class }\end{array}$ & \\
\hline \multicolumn{2}{|c|}{$\square$ properties } \\
\hline D & Data : byte[] \\
\hline $\mathrm{Ir}$ & InformationItems : List<InformationItem> \\
\hline L & Length : uint \\
\hline 里 & OverallResult : eOverallResult \\
\hline$P$ & ParentFieldNumber : uint \\
\hline$P$ & ParentRecordType : uint \\
\hline$R$ & Results : List<Result > \\
\hline \multicolumn{2}{|c|}{$\square$ Methods } \\
\hline$=9$ & SubField () (+ 2 overloads) \\
\hline$=\mathrm{T}$ & ToString 0 : string \\
\hline$=\mathrm{T}$ & ToStringErrorsOnly 0 : string \\
\hline
\end{tabular}

Figure 2 12: “Field" and "Subfield" Classes

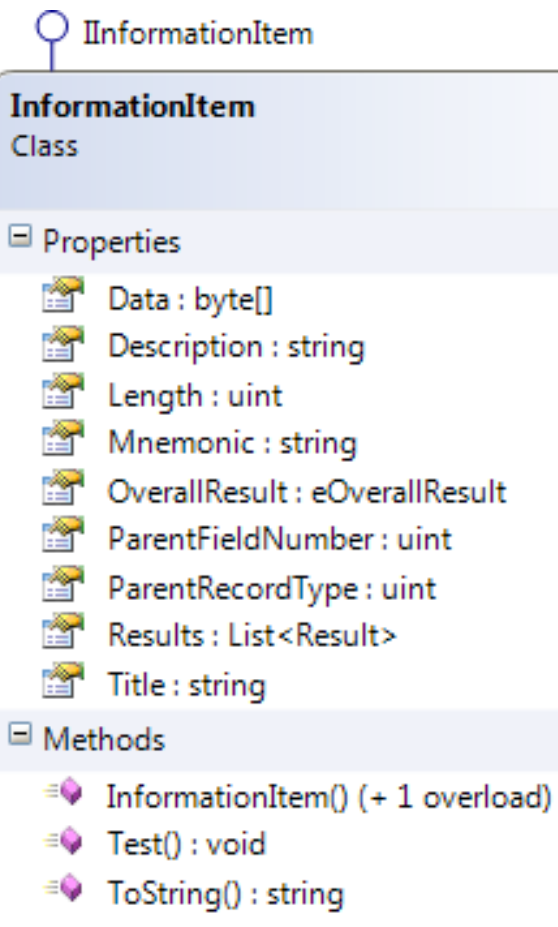
BinarySubField
Class
$\rightarrow$ SubField
$\square$ Properties
圅 Description : string

Figure 2 13: "InformastionItem" and "BinarySubField" Classes 
A Record is made up of Fields, and every Record inherits from a base Record class. As shown in Fig. 2-14 Binary Records, such as Types 4, 7 and 8 inherit from a Subclass of Record - the BinaryRecord class.

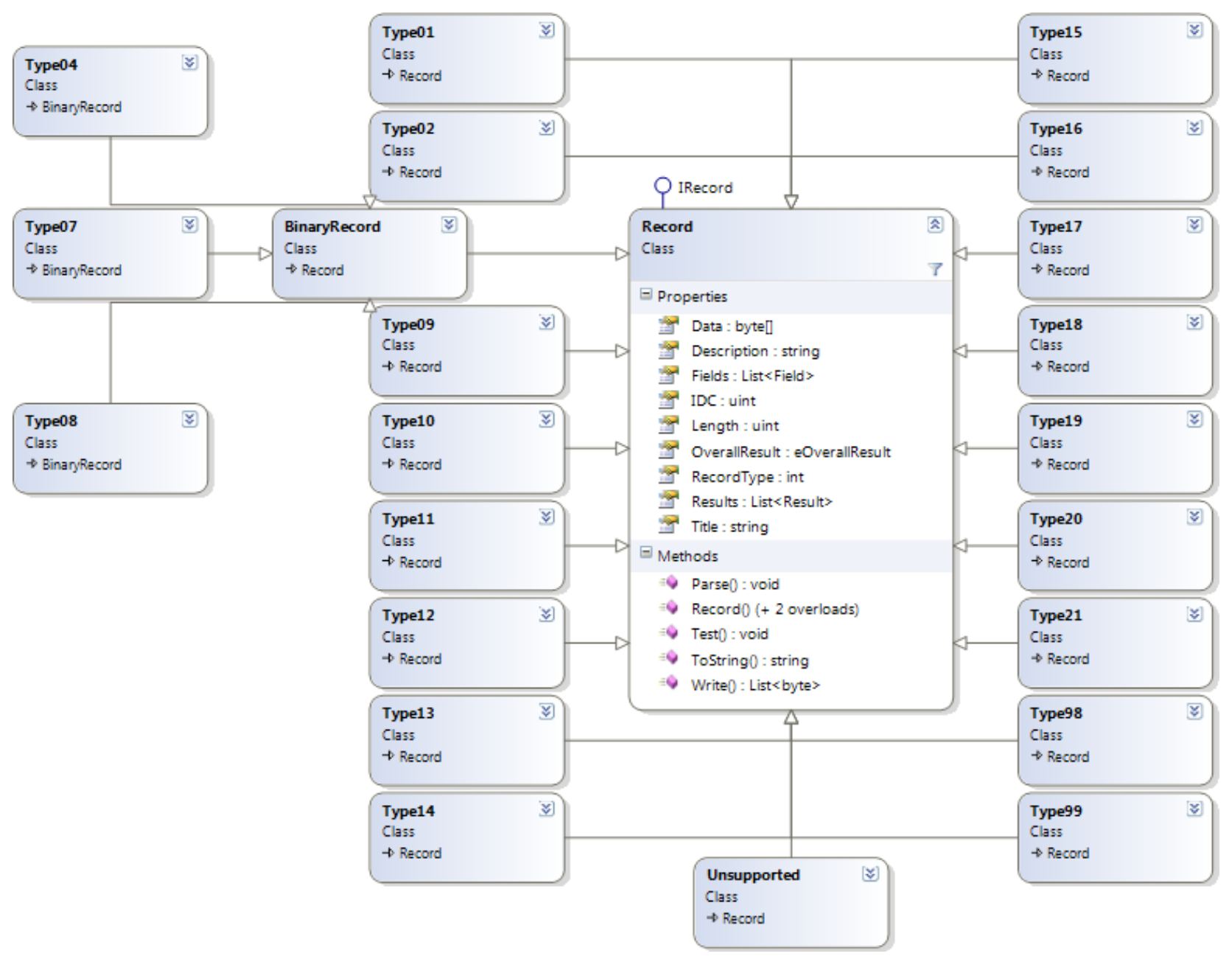

Figure 2-14: "Record” Class and Inheritance

\subsubsection{Parsing}

\subsubsection{Pre-Parse}

Two major operations occur during Pre-Parsing: (a) determining if the File Path passed into the software exists (e.g. a valid file), and (b) creating a Table of Contents (TOC) for the Transaction (by parsing of fields 1.001 to 1.003 ).

Field 1.001 is the length of the Type-1 Record and Field 1.003 describes the other record types within the transaction. After getting these two bits of information, the Pre-Parser skips to each record's first field to record their length. The end result is a TOC where each entry has a Record Type Number, IDC Value, and Record Length (in bytes). 
After these two operations are completed, a test to ensure that the summation of Table of Contents' length values is equal to the total number of bytes in the file is performed. The pseudo-code below describes the Pre-Parse program flow.

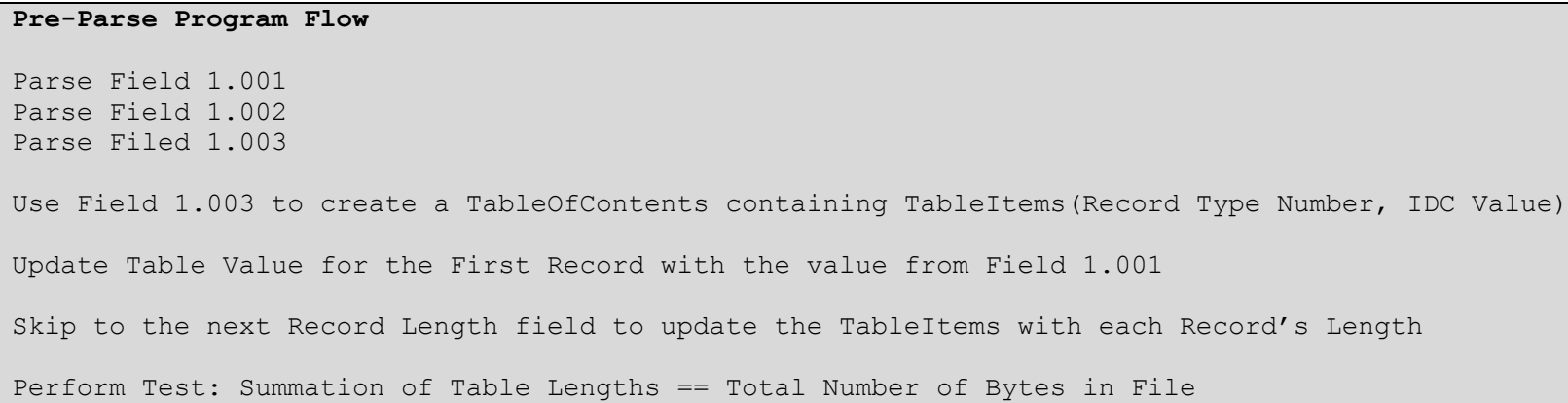

\subsubsection{Parse Fields and Records}

After Pre-Parsing is complete, the types and lengths of all Records in the Transaction are known. The Transaction data is split according to each Record's specified length, creating a list of records (List $<$ Record $>$ ). Each Record is passed a portion of the Transaction's bytes, which it uses to create a list of fields (List<Field>). Each Field has a list of Subfields (List<Subfield $>$ ), and each Subfield has a list of Information Items (List<Information Item $>$ ). The following pseudo-code describes this program flow.

Note: Every field is parsed as having subfields with information items, even fields that ANSI/NISTITL 1-2011 specifies as having no subfields and fields that ANSI/NIST-ITL 1-2011 specifies as having subfields which contain no information items. This intentional change was made for parsing consistency and because of the observation that there is no difference in encoding between a field with one subfield or information item and a field containing simply data.

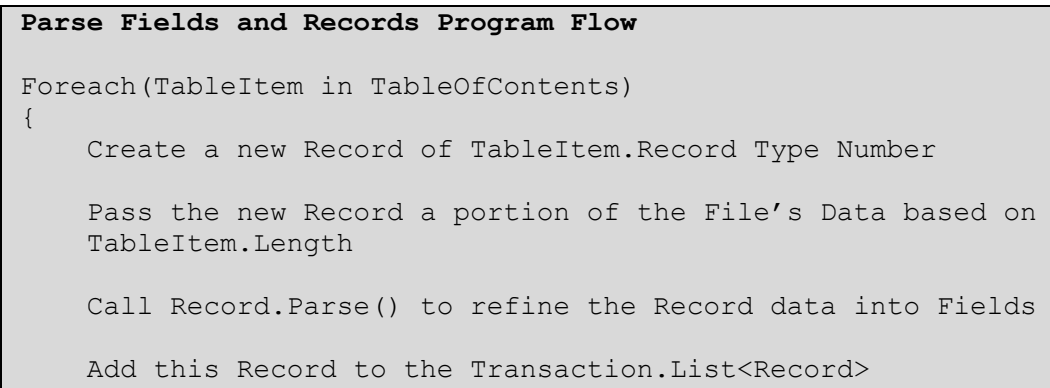

\subsubsection{Testing}

Testing can begin only after parsing is successfully completed. Testing is performed in isolation when possible following this approach:

- Transaction-Level Testing: Needs information from more than one Record 
- Record-Level Testing: Needs information from more than one Field within the same Record

- Field-Level Testing: Needs no more information than what is stored within a single Field

\subsubsection{Transaction-Level Testing - (AN2K11. Test ())}

This method performs two operations:

- Loop over the Transaction's list of Records calling Record. Test () on each in turn.

- Transaction-based Level-2 testing requires information from more than one Record.

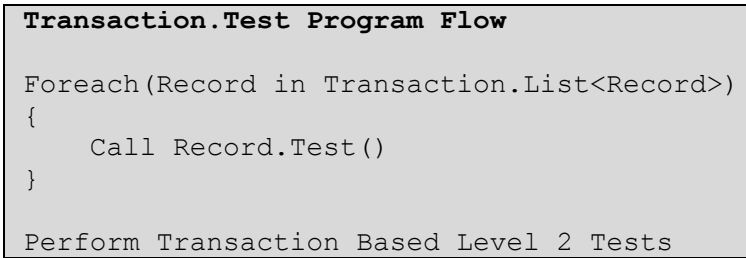

\subsubsection{Record-Level Testing-(Record.Test ())}

This method performs two operations:

- Loop over the Record's list of Fields calling Field. Test () on each in turn.

- Record-based Level-2 testing requires information from more than one Field within the Record.

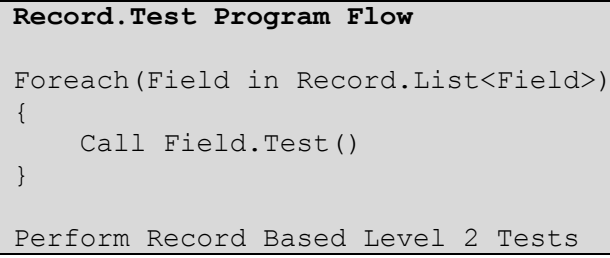

\subsubsection{Field-Level Testing - (Field.Test())}

This method performs Level-1 testing on the Field's Subfields and Information Items, ensuring correct count number, lengths, and value tests, etc. where applicable.

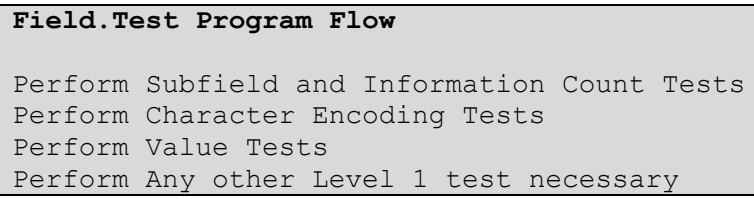

The BioCTS 2012 for AN-2011 High-Level Architecture depicting Pre-Parsing, Parsing, and Testing operations is shown in Fig. 2-15. The operations described above from Pre-parsing to Field-level testing are depicted. The test results are provided in two formats: text format and XML to facilitate automated analysis of test results when a large number of transactions are tested. 


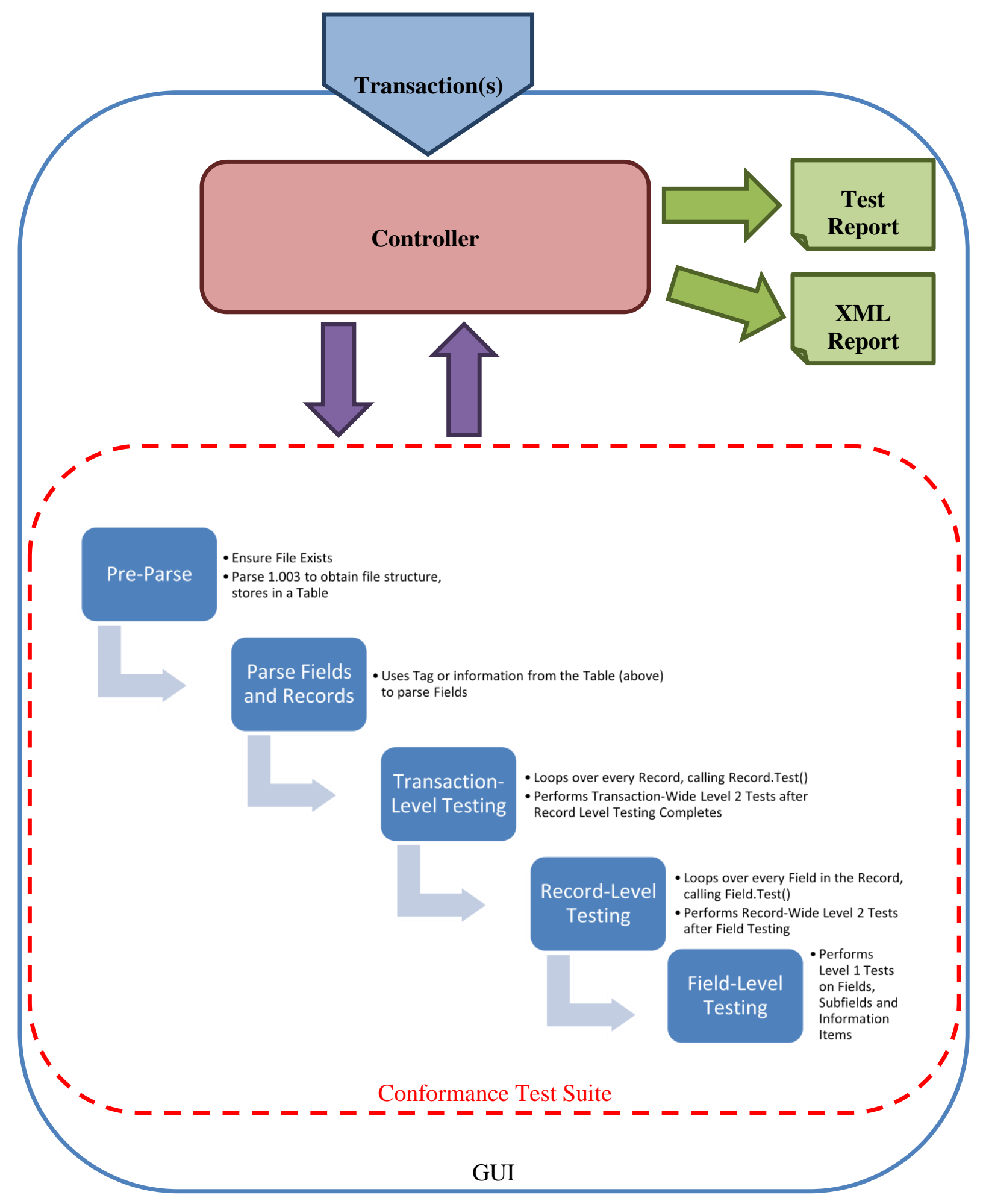

Figure 2 15: BioCTS 2012 for AN-2011 High-Level Architecture 


\subsubsection{Clarifying AN-2011 Field Structure Definitions}

The following Field structures are contained in ANSI/NIST ITL 1-2011, but a formal definition (including the underlying subfield and information item organization) is not provided:

- Single Info Item

- Shown in tables as a Field containing data

- Multiple Info Items

○ Shown in tables as a Field containing multiple Info Items

- Subfields: Repeating Sets of Info Items

- Shown in tables as a Field containing one or more Subfields which each contain multiple information items

- Subfields Repeating Values

○ "Shown in tables as a Field containing Subfields which contain data"

The difficulty in parsing and defining the Subfield and Information Items structures comes from the following observation:

It is impossible to differentiate between a single subfield, a single information item, and a single piece of data in the Traditional Encoding defined in Annex B of AN-2011.

Information Items are defined as the information separated by the ASCII Unit Separator character $\left({ }_{\mathrm{S}}\right)$. The number of Information Items present $\left(\mathrm{N}_{\mathrm{ii}}\right)$ equals the number of ${ }_{\mathrm{S}}$ separators present $\left(\mathrm{N}_{\mathrm{us}}\right)$ plus one: $\mathrm{N}_{\mathrm{ii}}=\mathrm{N}_{\mathrm{us}}+1$. Therefore, when there is a single Information Item present, there are no Unit Separators present $\left(\mathrm{N}_{\mathrm{us}}=0\right)$. Similarly, the number of Subfields present $\left(\mathrm{N}_{\mathrm{sf}}\right)$ equals the number of ASCII Record Separator characters $\left({ }^{\mathrm{R}}{ }_{\mathrm{s}}\right)$ present $\left(\mathrm{N}_{\mathrm{rs}}\right)$ plus one: $\mathrm{N}_{\mathrm{sf}}=\mathrm{N}_{\mathrm{rs}}+1$. Therefore, when there is a single Subfield present, there are no Record Separators present $\left(\mathrm{N}_{\mathrm{rs}}=0\right)$. Because there are no separators present in these cases, there is no difference between single instances of Subfields, Information Items, and data. The ${ }_{\mathrm{S}}$ character is used to identify the end of a field except when that field is the last field in the record; in this case the ${ }_{\mathrm{S}}^{\mathrm{S}}$ character is used.

To simplify parsing, and to be able to treat every field the same, BioCTS 2012 AN-2011 defines every field as having a list of subfields with each subfield containing a list of information items. The program flow describing Tagged Field parsing in shown below:

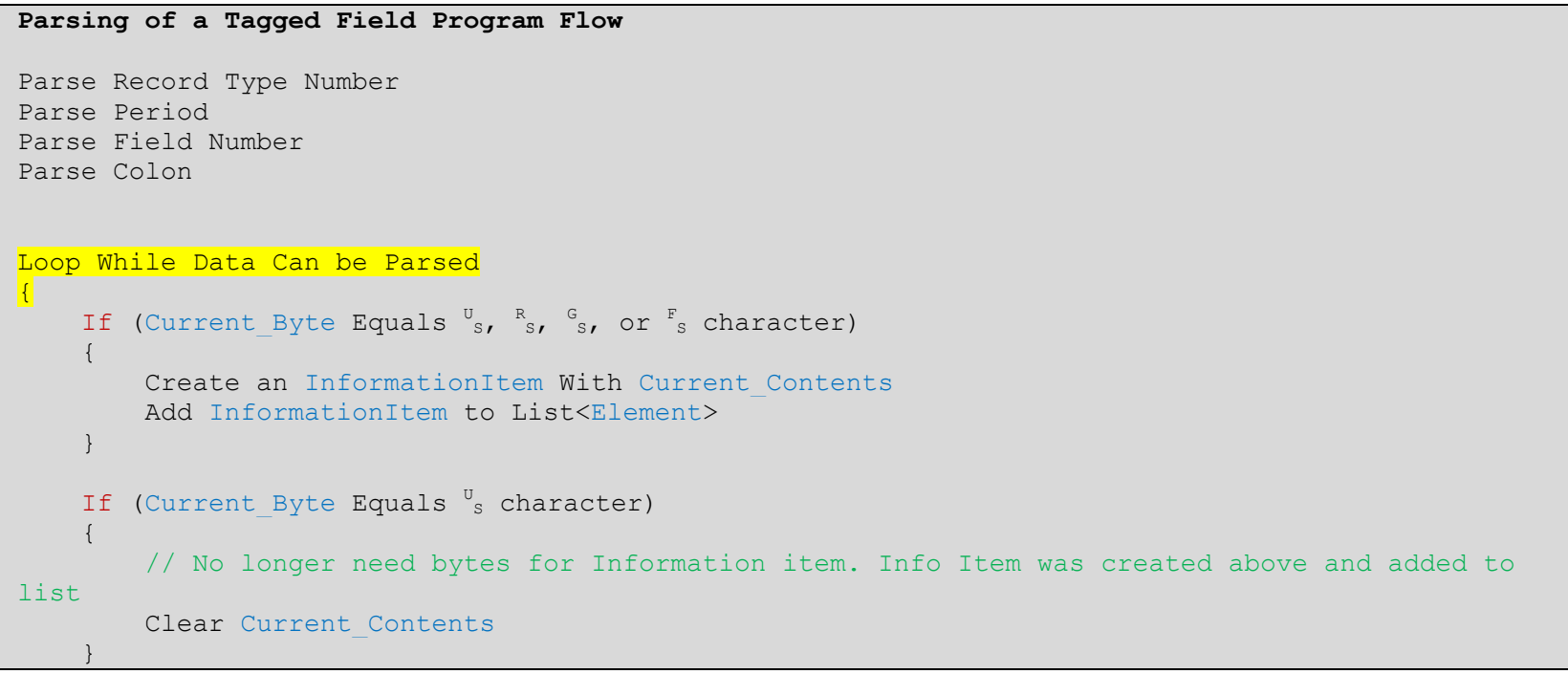




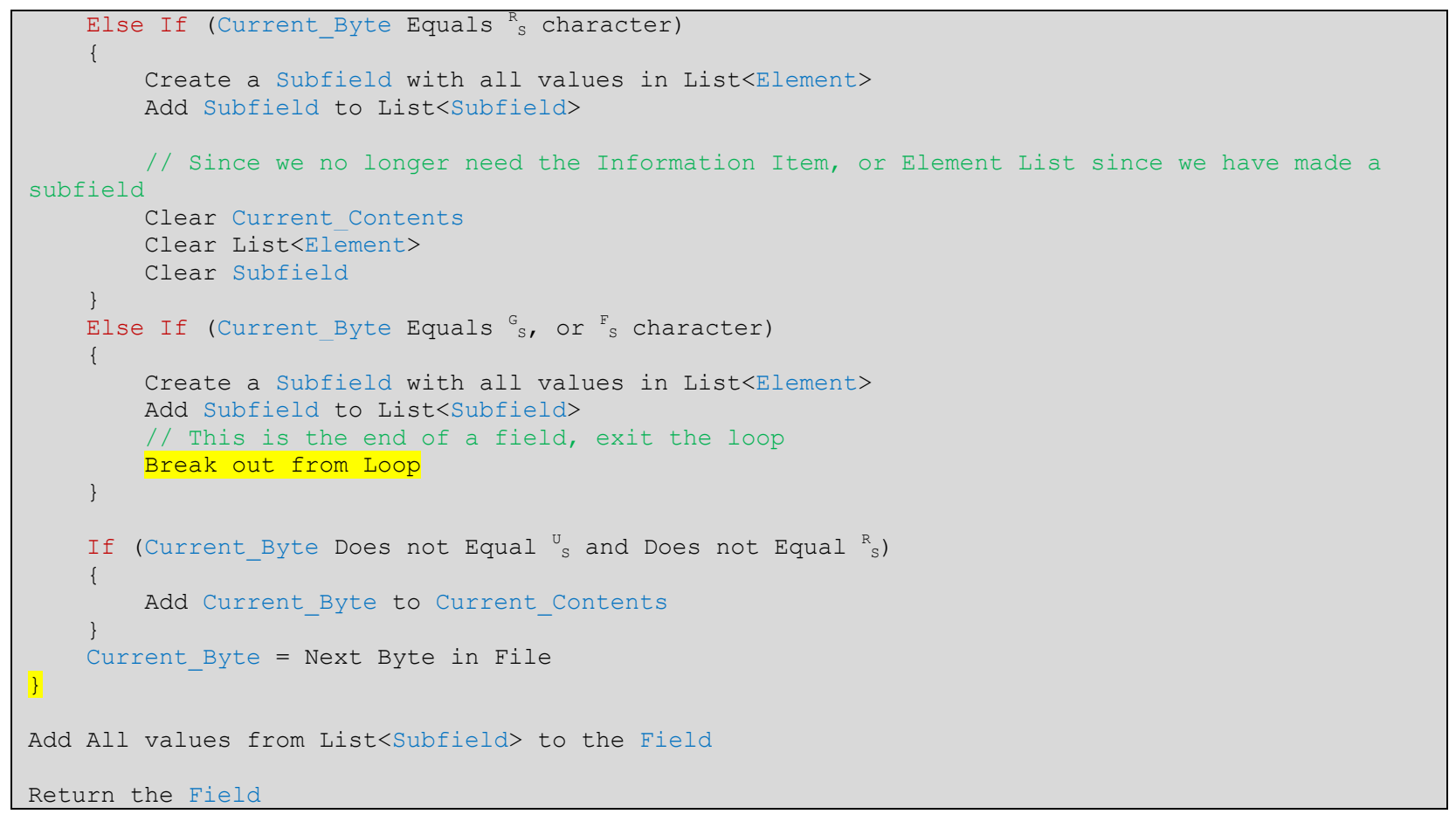

Table 2-1 compares how the field structure is specified in the standard and how it is defined in the code. In general, the standard does not mention Subfields or Information Items (although they exist) when there is only one instance. The following nomenclature is used in the table:

FN: Field Number, including record type.

II: Information Item. A single piece of information (data).

SF: Subfield. A set of one or more information items. (when more than one set is present)

\begin{tabular}{|c|c|c|}
\hline Description & Field Structure in Standard & Field Structure In Code \\
\hline $\begin{array}{l}\text { Single Information } \\
\text { Item } \\
\text { (A Field with Data) }\end{array}$ & $\begin{array}{l}\text { FN: } \text { II }^{\mathrm{G}}{ }_{\mathrm{S}} \\
\text { Note: the standard does not mention a subfield or } \\
\text { information item for this structure, but simply refers to } \\
\text { the "field" and its "data". }\end{array}$ & $\begin{array}{l}\text { Field contains one subfield that } \\
\text { contains one information item. } \\
\text { Data testing results reported at the } \\
\text { field level because it is viewed as a } \\
\text { field with data. }\end{array}$ \\
\hline $\begin{array}{l}\text { Multiple Information } \\
\text { Items } \\
\text { (A Field with } \\
\text { multiple Information } \\
\text { Items) }\end{array}$ & $\begin{array}{l}\text { FN: } \mathbf{I I}_{\mathbf{a}} \mathrm{U}_{\mathrm{S}} \ldots \mathbf{I I}_{\mathbf{n}} \mathrm{G}_{\mathrm{S}} \\
\text { Where } \mathrm{n} \text { is the number of information items } \\
\text { Note: the standard does not mention a subfield, just } \\
\text { information items. }\end{array}$ & $\begin{array}{l}\text { Field contains one subfield that } \\
\text { contains multiple information } \\
\text { items. } \\
\text { Data testing results reported at the } \\
\text { information item level because it is } \\
\text { viewed as a field that contains } \\
\text { several information items. }\end{array}$ \\
\hline $\begin{array}{l}\text { Subfields: } \\
\text { Repeating Sets Of } \\
\text { Information Items }\end{array}$ & $\begin{array}{l}\text { If subfield count }>1 \text { : } \\
\text { FN: } \mathbf{I I}_{\mathbf{1 a}} \mathrm{U}_{\mathrm{S}} \ldots \mathbf{I I}_{\mathbf{1 j}}{ }_{\mathrm{S}} \mathbf{I I}_{\mathbf{n a}} \mathrm{U}_{\mathrm{S}} \ldots \mathbf{I I}_{\mathbf{n k}} \mathrm{G}_{\mathrm{S}} \\
\text { Where } \mathrm{n} \text { is the number of subfields } \\
\text { If subfield count }=1 \text { : } \\
\mathbf{F N}: \mathbf{I I}_{\mathbf{a}} \mathrm{U}_{\mathrm{U}} \ldots \mathbf{I I}_{\mathbf{j}} \mathrm{G}_{\mathrm{S}}\end{array}$ & $\begin{array}{l}\text { Field contains one or more } \\
\text { subfields and each subfield contains } \\
\text { multiple information items. } \\
\text { Data testing results reported at the }\end{array}$ \\
\hline
\end{tabular}




\begin{tabular}{|c|l|l|}
\hline & & $\begin{array}{l}\text { information item level because } \\
\text { each subfield contains several } \\
\text { information items. }\end{array}$ \\
\hline $\begin{array}{c}\text { Subfields: } \\
\text { Repeating Values }\end{array}$ & $\begin{array}{l}\text { If subfield count }>1 \text { : } \\
\text { FN: } \mathbf{I I}_{\mathbf{a}} \mathrm{R} \\
\text { Where } \mathbf{n} \text { is the } \mathbf{I I}_{\mathbf{n}} \mathrm{G} \text { S } \\
\text { If subfield count }=1: \\
\text { FN: } \mathbf{I I}_{\mathbf{a}}^{\mathrm{G}} \mathrm{S}\end{array}$ & $\begin{array}{l}\text { Field contains one or more } \\
\text { subfields and each subfield contains } \\
\text { one information item. } \\
\text { Data testing results reported at the } \\
\text { subfield level because each subfield } \\
\text { contains a single information item. }\end{array}$ \\
\hline
\end{tabular}

Table 2-1: Comparison of the Field Structure in the Standard and the Code

As stated previously, it is impossible to differentiate between a single subfield, a single information item, and a single piece of data in the Traditional Encoding defined in Annex B of AN-2011.

Examples are shown below:

\begin{tabular}{|l|l|}
\hline Field contents & Actual encoding \\
\hline Data only & XX.YYY: $\langle\text { DATA }\rangle^{G}$ \\
\hline A single subfield & XX.YYY: $\langle\text { DATA }\rangle_{S}$ \\
\hline $\begin{array}{l}\text { A single subfield containing a single } \\
\text { information item }\end{array}$ & XX.YYY: $\langle\text { DATA }\rangle_{S}^{G}$ \\
\hline
\end{tabular}

Therefore, as shown in Fig.2-16, in BioCTS every field contains a list of subfields, which each contain a list of information items.

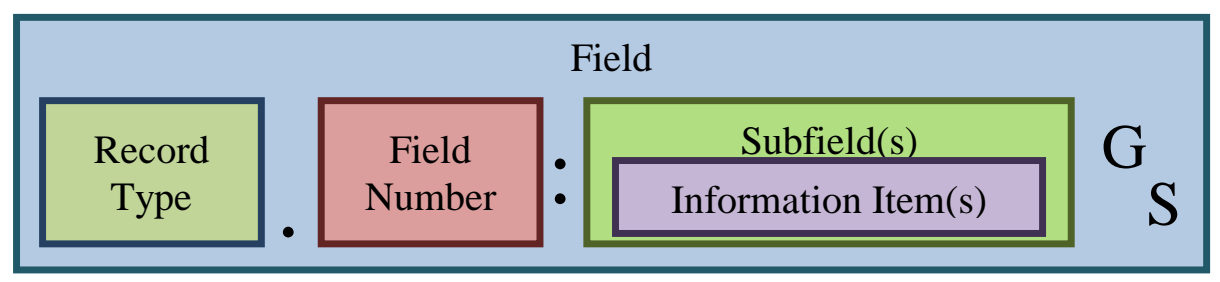

\section{Figure 2.16: Generic AN-2011 Field Structure}

The data for each field is held in the Information Item. However, results of tests can be stored at any level (Information Item, Subfield, Field, Record, and Transaction) as needed to align with the ANSI/NIST-ITL 1-2011 field definitions.

For example, a field defined with no subfields:

\begin{tabular}{|l|l|l|l|l|l|l|l|l|l}
\hline 1.002 & VER & VERSION NUMIRER & M & N & $\begin{array}{c}\mathrm{T}-4 ; \\
\mathrm{X}=3^{33}\end{array}$ & 4 & VER $=0500$ & 1 & 1 \\
\hline
\end{tabular}

$$
\text { Underlying structure: } 1.002: 0500^{G} \mathrm{~s}\left(\mathrm{FN}: \|^{G} \mathrm{~s}\right)
$$

Field 1.002 is represented in the software internally as shown in Fig. 2-17: 


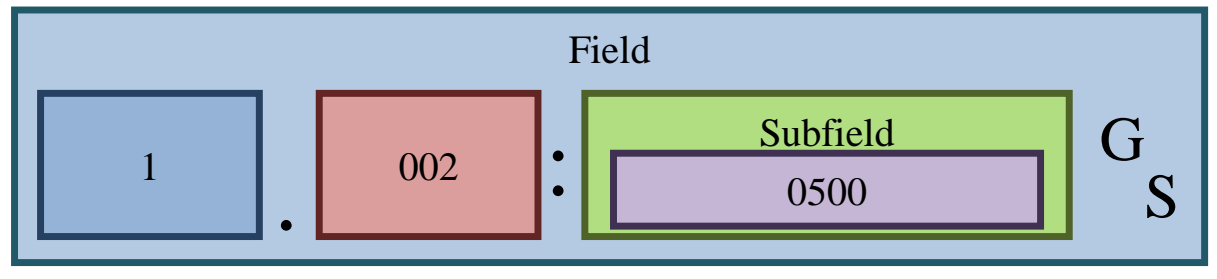

Figure 2.17: AN-2011 Field Structure Example

Field 1.002 is displayed in the CTA as:

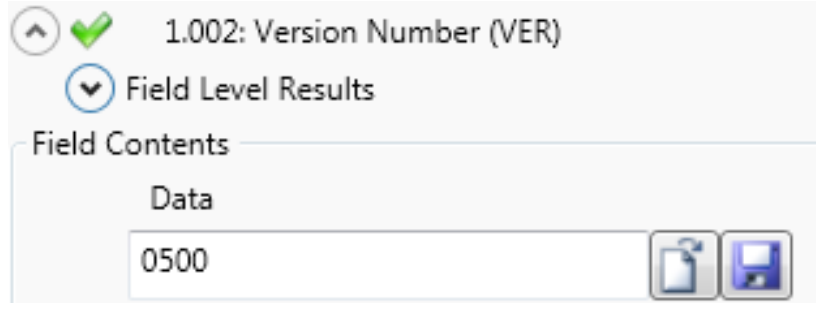

This matches the ANSI/NIST-ITL 1-2011 Field definition (note that it does not mention the Subfield or Information Item).

\subsubsection{BioCTS 2012 for AN-2011 Testing Exceptions}

Any tests implemented in code that differ from the requirements defined in the ANSI/NIST ITL2011, or any requirements untested are considered "exceptions". The CTS contains two types of test assertion exceptions: Record-Type Limitations and Requirement Limitations.

\subsubsection{Record Type Limitations}

The CTS provides parsing and IDC support for all Record Types in a given transaction and also tests for any deprecated or legacy values. However, only a selected subset of Record Types is fully supported and tested by the CTS:

- Type-1: Transaction Information

- Type-4: High-resolution grayscale fingerprint image

- Type-10: Face, other body part, or scar, mark tattoo (SMT) image

- Type-13: Variable-resolution latent friction ridge image

- Type-14: Variable-resolution fingerprint image

- Type-15: Variable-resolution palm print image

- Type-17: Iris image

Records in any Transaction that include Record Types other than the ones listed above are detected and their presence (including the location within the transaction) and length are reported in the test results.

In addition, some fields are supported for all Record Types due to structural requirements and interfield dependencies:

- Field xx.001-Record header. (All Record Types)

- Field xx.002-Information designation character / IDC (All Record Types)

- $\quad$ Field xx.016-Segments / SEG (Record Types 20 and 21) 
- Field xx.021-SRN/ACN (Record Types 20 and 21)

- Field xx.995-Associated Context / ASC (Record Types 10 and Above)

- Field xx.997-Source Representation / SOR (Record Types 10 and Above)

\subsubsection{Requirement Limitations:}

In some cases, testing a requirement was not possible without additional resources or information that was not obtained in time for inclusion in this version of the CTS:

- Domain Names/ Application Profile Specifications: requires referencing of evolving registrar databases. See ANSI/NIST ITL-2011 Sec. 5.3.2 and Sec. 6.

- Alternate Character Sets: Further research is required. Support for "user-defined" character set is not feasible. See ANSI/NIST ITL-2011 Sec. 5.6.

- Alternate Coordinate System: Further research required. Support for "user-defined" systems is not feasible. See ANSI/NIST ITL-2011 Sec. 7.7.3.

- Subject Acquisition Profiles: Some SAP/FAP/IAP level contain Level 3 requirements (image capture conditions). See ANSI/NIST ITL-2011 Sec. 7.7.5.

- ASEG-Open and Closed Paths for Polygons: Further research required. See ANSI/NIST ITL2011 Sec. 7.8. 


\section{References}

The list below provides references for this publication.

[1] ISO/IEC JTC 1/SC 37 Web page http://www.iso.org/iso/jtc1_sc37_home (accessed 8/20/12).

[2] ANSI/NIST-ITL Standard Web page http://www.nist.gov/itl/iad/ig/ansi_standard.cfm (accessed $8 / 20 / 12)$.

[3] F. L. Podio, D. Yaga, and C. J. McGinnis, Editors, NIST Special Publication 500-295, Conformance Testing Methodology for ANSI/NIST-ITL 1-2011, Data Format for the Interchange of Fingerprint, Facial \& Other Biometric Information (2012).

[4] Microsoft@ Download Center web page http://www.microsoft.com/en-us/download/default.aspx (accessed 8/27/12).

[5] P. Grother and W. Salamon, NIST Special Publication 800-76-2, Second Draft Biometric Data Specification for Personal Identity Verification, June 2012. 
Annex A - Roadmaps of Biometric Data Interchange Formats and Conformance Testing Methodologies

\section{A.1 First Generation of ISO/IEC Data Interchange Format Standards}

Figure A-1 depicts the first generation $(1 \mathrm{G})$ of biometric data interchange formats developed by ISO/IEC JTC 1/SC 37-Biometrics for a number of biometric modalities and their associated conformance testing methodology standards. The publication date of each of the standards is included. The development stage is indicated for the standards not yet published. The biometric standards of the first generation were specified in binary encoding as shown in the picture.

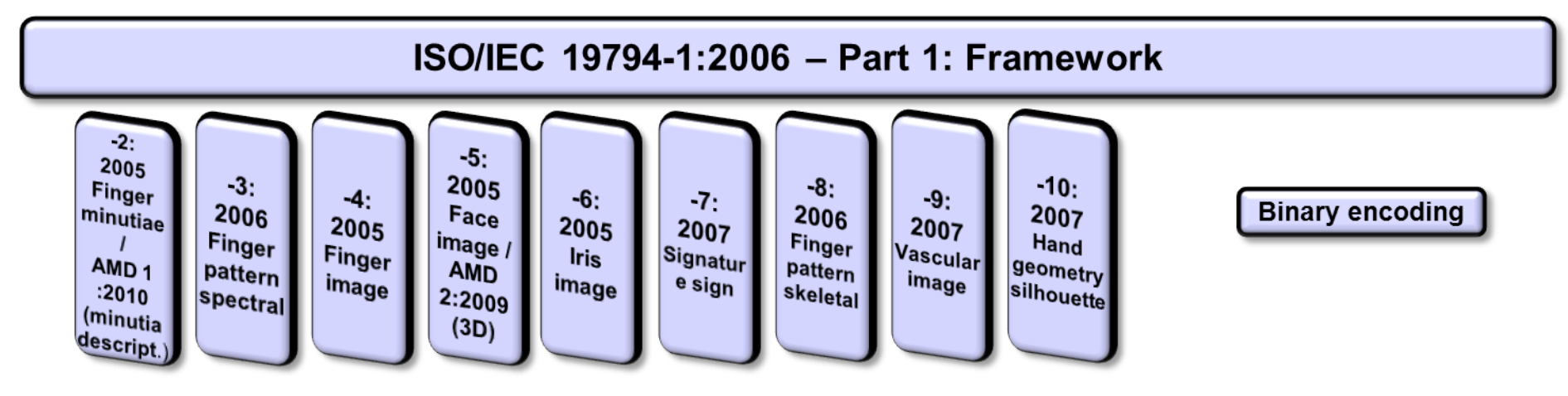

ISO/IEC 29109-1:2009 - Part 1: Generalized conformance testing methodology (CTM)
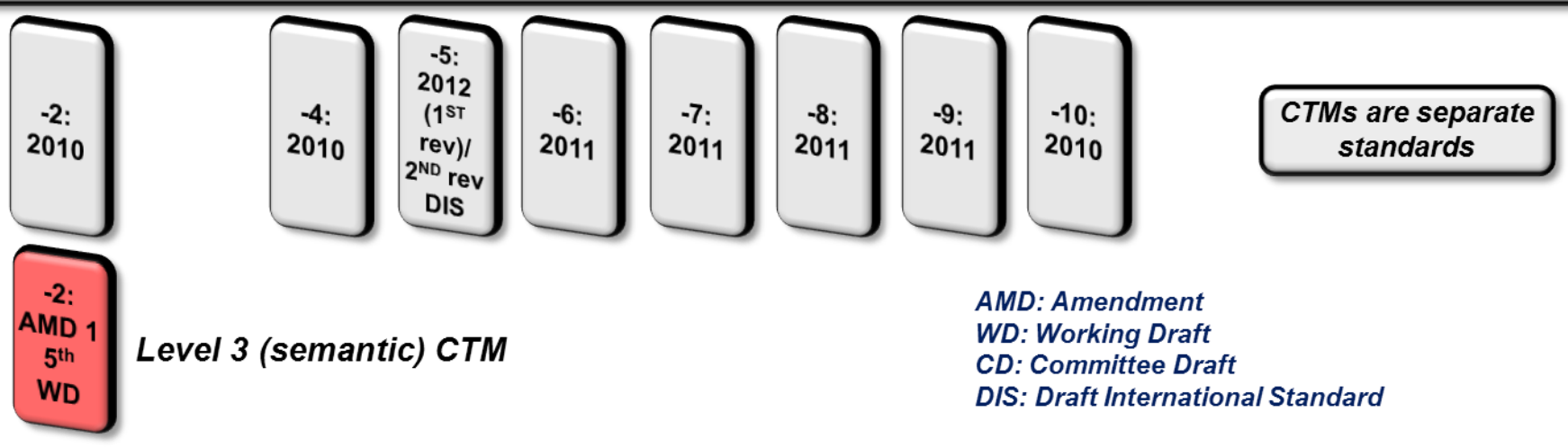

AMD: Amendment

WD: Working Draft

CD: Committee Draft

DIS: Draft International Standard

Figure A.1: 1G Biometric Data Formats and Associated CTMs 


\section{A.2 Second Generation of ISO/IEC Data Interchange Format Standards}

Figure A-2 depicts the same information as Figure A-1 but for the second generation (2G) of biometric data interchange format standards and associated CTM standards. Although the first generation of CTM standards are separate documents, the associated CTMs for the 2G data interchange formats are developed as Amendments to the data interchange format standards. A shown in the figure, most of the data interchange formats specified in binary encoding has been published. The Subcommittee is also developing XML encoding versions for a number of these standards.

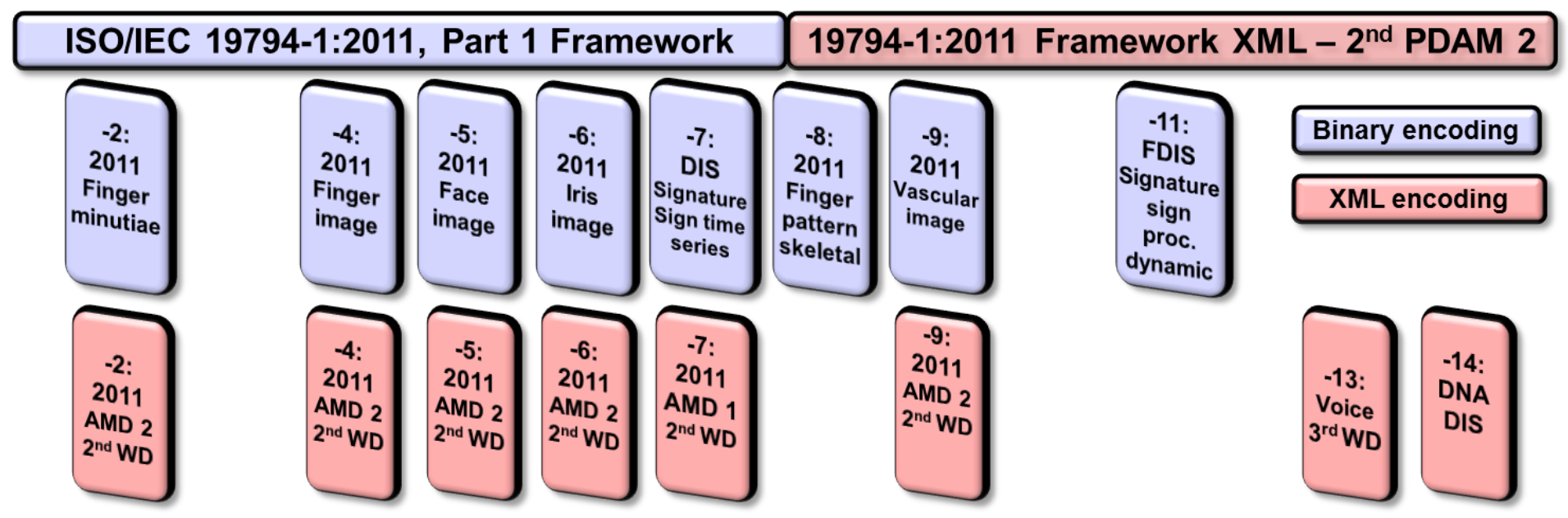

ISO/IEC 19794-1rev AMD 1 Conformance testing methodology (bin. encoding) - FDAM 1
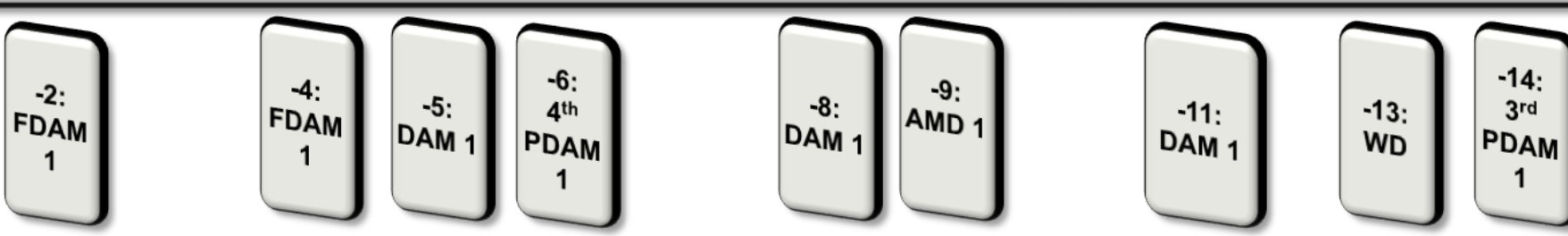

Figure A.2: 2G Biometric Data Formats and Associated CTMs 


\section{Annex B - Roadmaps of Conformance Test Tools for Biometric Data Interchange Formats}

\section{B.1 CTSs Designed to Test Implementations of AN-2011 Transactions}

Figure B.1 depicts the current roadmap of conformance tool development for AN-2011 Transactions. Plans are underway to expand the development of BioCTS 2012 to include testing of other Record Types. Currently, the test architecture supports testing of Transactions specified in Traditional Encoding. The roadmap includes extending the work to accommodate required testing of Transactions in the National Information Exchange Model (NIEM)-XML Encoding.

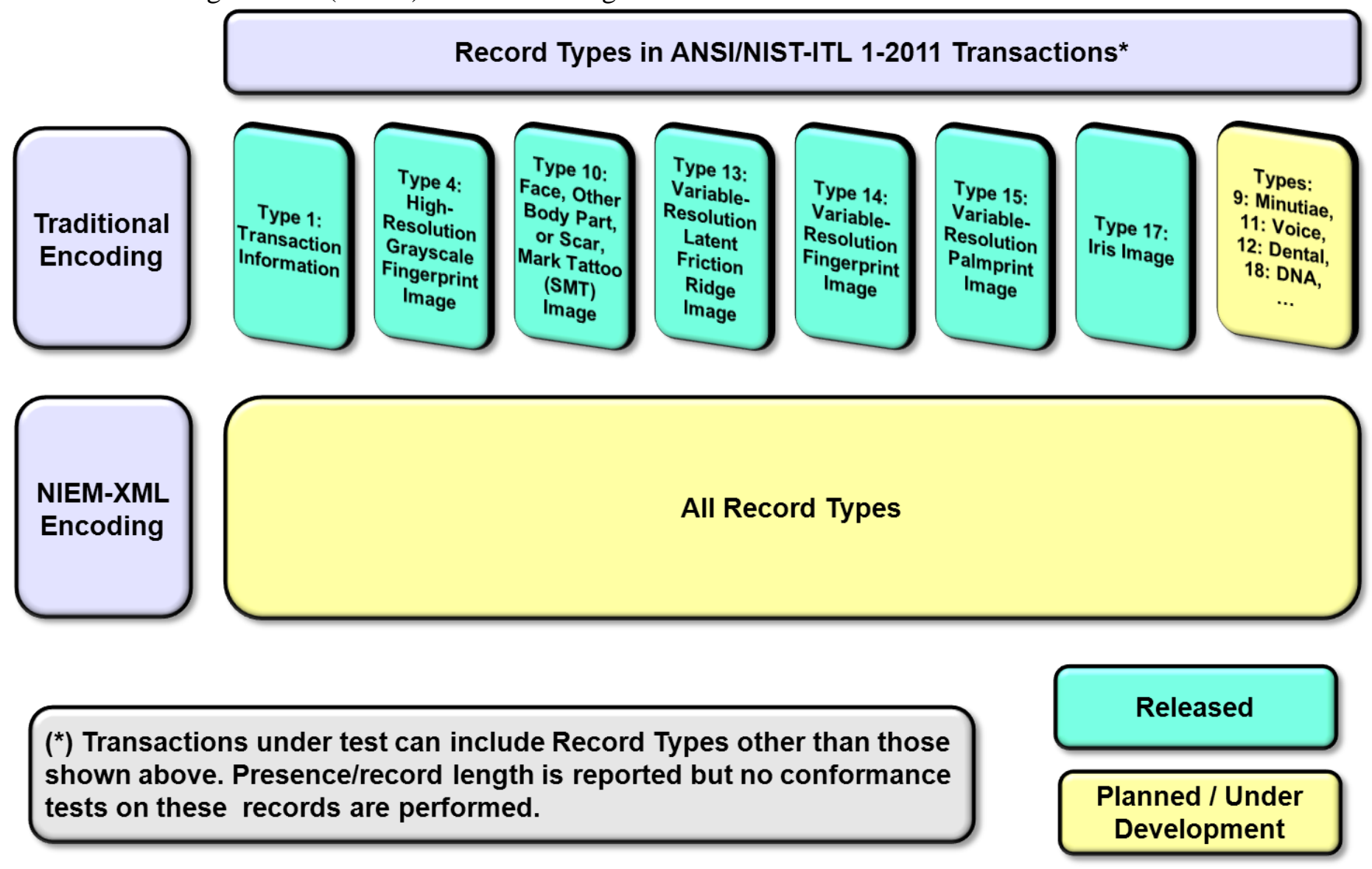

Figure B.1: Roadmap of CTSs for AN-2011 Transactions 


\section{B.2 CTSs Designed to Test Implementations of ISO/IEC Data Interchange Formats}

Figure B. 2 depicts the current roadmap for conformance test tools development for the ISO/IEC 19794-x biometric data interchange standards. CTSs designed to test implementations of the first generation of data formats were migrated this year to run under the advance conformance test architecture that is part of BioCTS 2012. Existing CTSs test binary encoding implementations. Further work will be focused on developing CTSs to test implementations of data formats for other modalities encoded in both binary and XML.

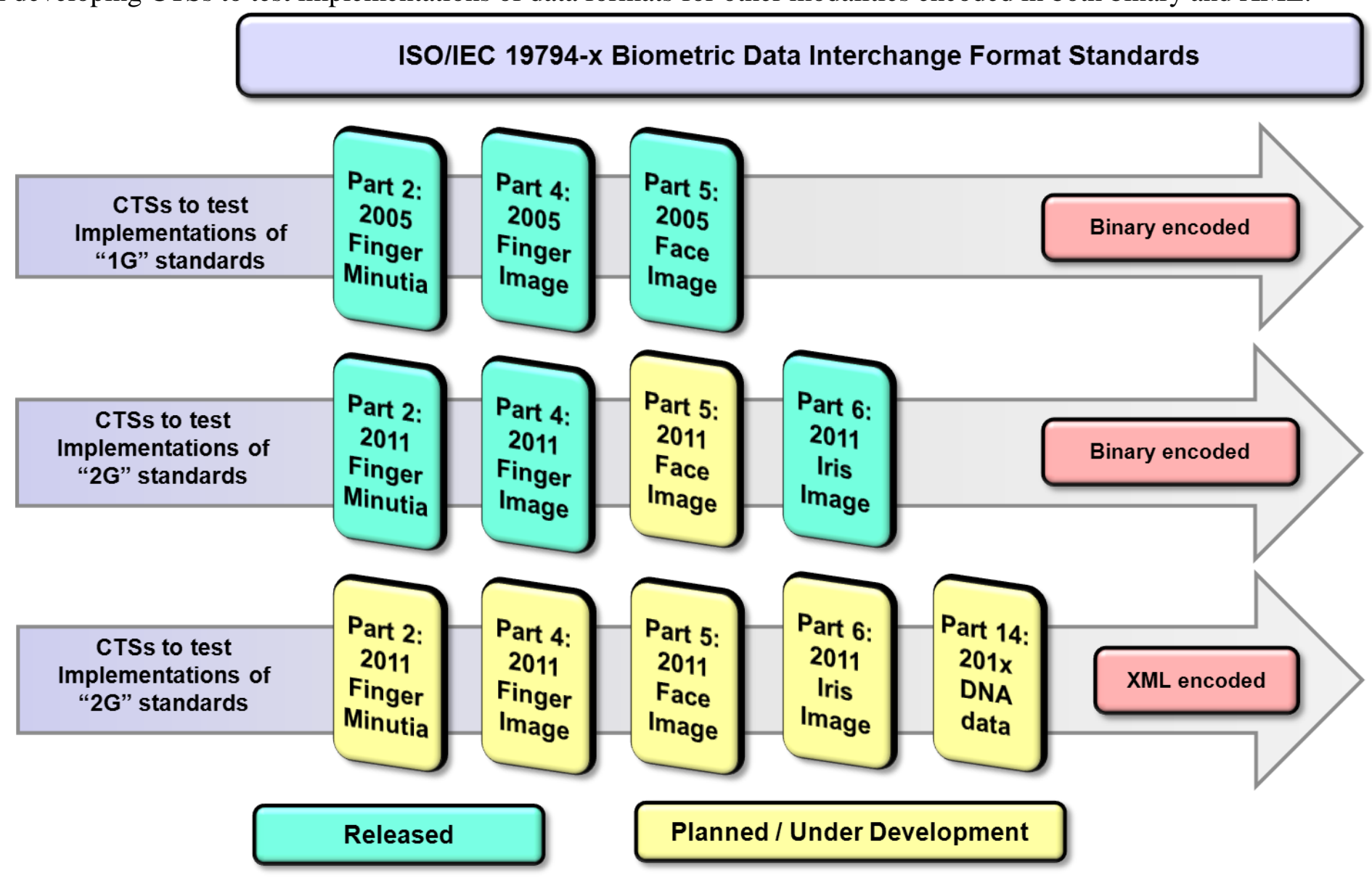

Figure B.2: Roadmap of CTSs for ISO/IEC Data Interchange Formats 


\section{B.3 CTS Designed to Test Implementations of PIV profiles specified in NIST SP 800-76-2}

Second Draft NIST Special Publication 800-76-2 was open for public comment until Noon on 4 August 15, 2012. Two CTSs were developed to test implementations of the ISO/IEC 19794-6:2011 profile. Additional CTSs are planned which will be designed to test implementations of national standards published by the American National Standards Institute (ANSI) and developed by Technical Committee M1 - Biometrics of the InterNational Committee for Information Technology Standards (INCITS). These profiles are also specified in NIST SP 800-76-2. Changes to the existing CTS will be made if technical changes to the ISO/IEC 19794-6:2001 profiles are made as a result of the public review.

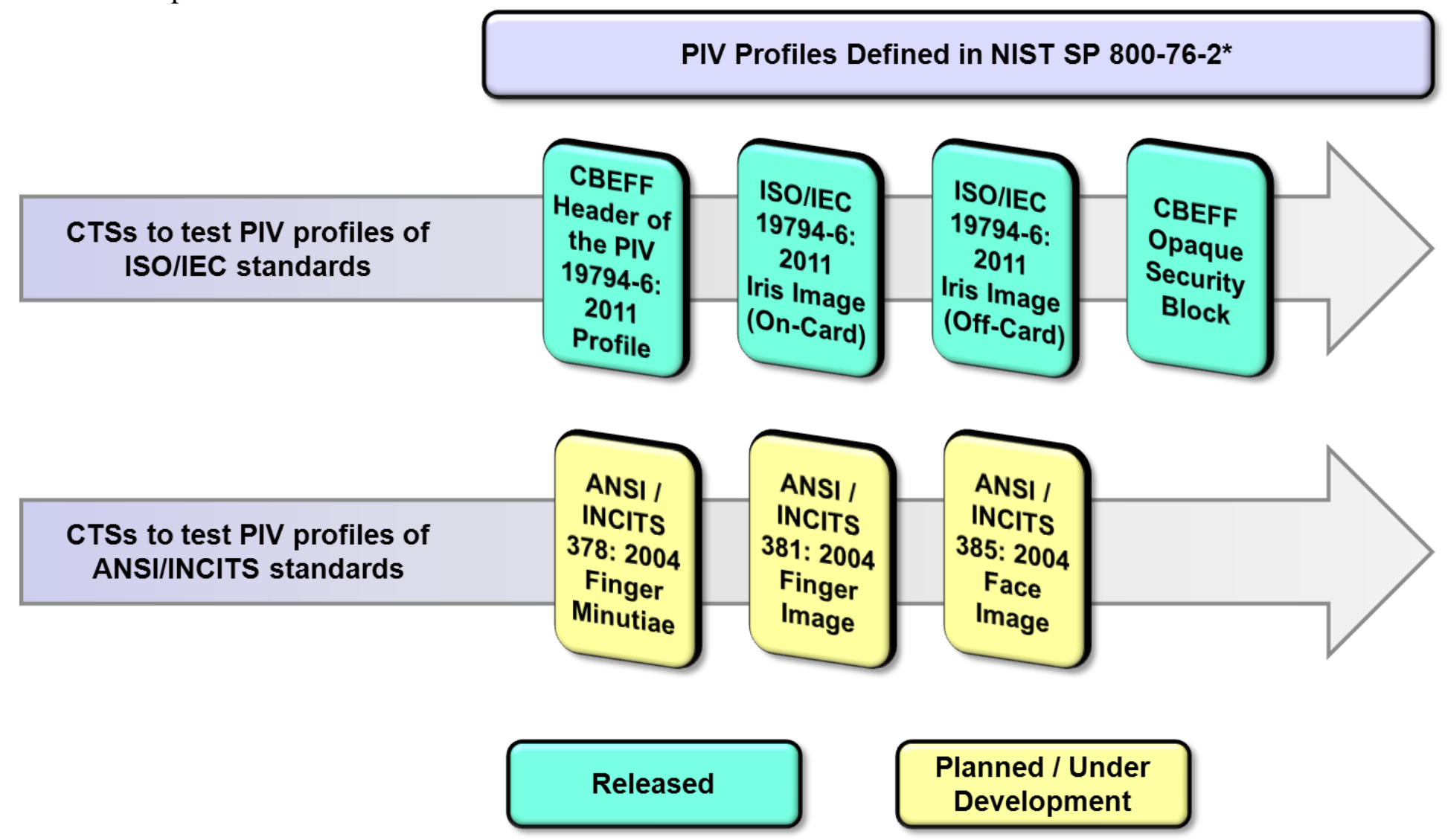

Figure B.3: Roadmap of CTSs for PIV Profiles Defined in NIST SP 800-76-2 